\title{
YiQiFuMai Powder Injection Ameliorates Cerebral Ischemia by Inhibiting Endoplasmic Reticulum Stress-Mediated Neuronal Apoptosis
}

\author{
Guosheng Cao, Huana Zhou, Nan Jiang, Yuwei Han, Yang Hu, \\ Yuanyuan Zhang, Jin Qi, Junping Kou, and Boyang Yu \\ Jiangsu Key Laboratory of TCM Evaluation and Translational Research, Department of Complex Prescription of TCM, \\ China Pharmaceutical University, Nanjing 211198, China
}

Correspondence should be addressed to Junping Kou; junpingkou@cpu.edu.cn

Received 30 November 2015; Accepted 4 February 2016

Academic Editor: Giuseppe Cirillo

Copyright (C) 2016 Guosheng Cao et al. This is an open access article distributed under the Creative Commons Attribution License, which permits unrestricted use, distribution, and reproduction in any medium, provided the original work is properly cited.

YiQiFuMai (YQFM) powder injection as a modern preparation derived from Sheng Mai San, a traditional Chinese medicine, has been widely used in the treatment of cardiovascular and cerebrovascular diseases. However, its neuroprotective effect and underlying mechanism in cerebral ischemia remain to be explored. The present study was designed to investigate the neuroprotective effect of YQFM on endoplasmic reticulum (ER) stress-mediated neuronal apoptosis in the permanent middle cerebral artery occlusion- (MCAO-) injured mice and the oxygen-glucose deprivation- (OGD-) induced pheochromocytoma (PC12) cells. The results showed that single administration of YQFM $(1.342 \mathrm{~g} / \mathrm{kg}$, i.p.) could reduce the brain infarction and improve the neurological deficits and the cerebral blood flow (CBF) after MCAO for $24 \mathrm{~h}$ in mice. Moreover, incubation with YQFM (100, 200 , and $400 \mu \mathrm{g} / \mathrm{mL}$ ) could increase the cell viability, decrease the caspase-3 activity, and inhibit the cell apoptosis in OGD-induced PC12 cells for $12 \mathrm{~h}$. In addition, YQFM treatment could significantly modulate cleaved caspase-3 and Bcl-2 expressions and inhibit the expressions of ER stress-related marker proteins and signaling pathways in vivo and in vitro. In conclusion, our findings provide the first evidence that YQFM ameliorates cerebral ischemic injury linked with modulating ER stress-related signaling pathways, which provided some new insights for its prevention and treatment of cerebral ischemia diseases.

\section{Introduction}

Ischemic stroke is a devastating disease that ranks secondly after cardiac ischemia as a cause of death and permanent disability worldwide, estimated by the World Health Organization [1]. Since a series of complexed mechanisms are involved in the pathology [2], investigations on the specific and potent treatment for the neuronal functional recovery after cerebral ischemic stroke were extremely limited [3]. According to recent studies, endoplasmic reticulum (ER) stress is one of the essential signaling mechanisms during neuronal injury resulting from cerebral ischemia [4-7]. In response to ischemia or oxygen-glucose deprivation (OGD),
ER appears swollen, leading to calcium ion disorder and cell apoptosis through caspase-12 activation [8, 9].

Glucose-regulated protein 78 (GRP78) is a main ER molecular chaperone, regulating protein folding and facilitating protein translocation and protein secretion in ER. It is widely used as a sentinel marker for ER stress under pathologic conditions [10]. C/EBP homologous protein (CHOP) is the first protein identified that promotes apoptosis when ER stress happens. And it is reported to induce apoptosis through downregulating the antiapoptotic factor, Bcl-2 [11]. Activating transcription factor-4 (ATF4) is a stress responsive gene, which is also known as an important mediator of the unfolded protein response (UPR). When ATF4 is 
ubiquitously expressed at low basal level, its initiation of translation at the authentic start codon is dependent on the upregulated phosphorylation of eIF2 $\alpha$ by ER stress [12]. Moreover, the expressions of ER stress-target genes, such as $\mathrm{CHOP}$ and $\mathrm{X}$-box-binding protein-1 (XBP-1), are also modulated by an ER-membrane-bound transcription factor, activating transcription factor-6 (ATF-6) $[13,14]$. Abundant evidence has shown that the inhibition of all three ER stressrelated pathways and the cell apoptosis exerts a remarkable protective effect on cerebral ischemia and therefore might be a novel and effective treatment after ischemic stroke $[8,15,16]$.

YiQiFuMai (YQFM) powder injection is a modern preparation based on a well-known complex prescription, Sheng Mai San, which is composed of Panax ginseng C. A. Mey, Ophiopogon japonicus (Thunb.) Ker-Gawl., and Schisandra chinensis (Turcz.) Baill. (1:3:1.5). It was approved in 2007 for treatment of microcirculatory disturbance-related diseases by the China Food and Drug Administration in China [17]. Pharmacological studies have demonstrated that YQFM exerts its beneficial effects on myocardial, vascular, or intestine injuries mainly through inhibiting oxidative damage, NF- $\kappa \mathrm{B}$ activation, and cytokines expressions [1719]. In addition, our network pharmacological studies have also indicated that multiple antiapoptotic pathways and ER stress-related signaling pathways are possibly involved in the mechanism of YQFM against cardiocerebral ischemia diseases [20]. Furthermore, YQFM has been confirmed to ameliorate blood-brain barrier (BBB) dysfunction and brain edema induced by focal cerebral ischemia-reperfusion in mice [21]. However, the effect and potential mechanisms of YQFM on the neuronal apoptosis or oxidative injury remain to be explored.

Given the key role of ER stress-mediated neuronal apoptosis in ischemic stroke [6], the present study is designed to further verify the protective effect of YQFM in permanent middle cerebral artery occlusion- (MCAO-) injured mice and OGD-induced differentiated rat pheochromocytoma (PC12) cells and to explore the involvement of ER stress-related signaling pathways in its effect on neuronal apoptosis. Our results would confirm the efficacy of YQFM in ischemic stroke and may provide some novel insights for its prevention and treatment of cerebral ischemia diseases.

\section{Materials and Methods}

2.1. Reagents. YQFM was purchased from Tasly Pharmaceutical Co., Ltd. (Tianjin, China, batch number 20121210). Dantrolene (Dan) was purchased from Sigma (St. Louis, MO, USA). Hoechst 33342 (bisbenzimide) and enhanced chemiluminescence (ECL) reagents were obtained from Beyotime Biotechnology (Shanghai, China). Polyvinylidene fluoride (PVDF) membranes were purchased from Millipore (Bedford, MA, USA). 3-(4,5-Dimethylthiazol-2-yl)-2,5diphenyltetrazolium bromide (MTT) was purchased from Ameresco (Ameresco, OH, USA). The primary antibodies, horseradish peroxidase- (HRP-) conjugated goat anti-rabbit and anti-mouse IgG, were purchased from Bioworld Technology, Inc. (St. Louis Park, MN, USA).
2.2. Animals. Male C57BL/6J mice weighing 18-22 g were obtained from the Model Animal Research Centre of Yangzhou University (Yangzhou, China, certificate number SCXK 2014-0004). All procedures and assessments were approved by the Animal Ethics Committee of the School of Chinese Materia Medica, China Pharmaceutical University. The experiments were carried out in accordance with the National Institutes of Health Guide for the care and use of laboratory animals (NIH Publication number 80-23, revised 1996). Mice were housed in a $12 \mathrm{~h}$ light-dark cycle and allowed free access to food and water. Prior to experiments, all animals were randomized into experimental groups and measured blindly.

2.3. Focal Cerebral Ischemia. C57BL/6J mice were subjected to MCAO as reported previously [22]. Briefly, animals were anesthetized with $4 \%$ chloral hydrate $(0.1 \mathrm{~mL} / 10 \mathrm{~g}$ body weight) intraperitoneally (i.p.), after a midline incision of the neck skin, and branches of the right external carotid artery were isolated and cauterized. In brief, a 6-0 nylon monofilament suture, blunted at the tip and coated with $1 \%$ poly-L-lysine, was advanced $9-10 \mathrm{~mm}$ into the internal carotid to occlude the origin of the middle cerebral artery (MCA) for $24 \mathrm{~h}$. Body temperature was maintained with a heating pad (Alcbio; Shanghai, China) at $37.0 \pm 0.5^{\circ} \mathrm{C}$ during operation and ischemia. Sham-operated animals underwent the same surgical procedure, but the suture was not advanced into the internal carotid artery. A laser Doppler flow meter (LDF; FLPI2, Moor, England) was used to confirm the decrease of the blood flow immediately after the occlusion to below $30 \%$ compared with nonischemia side.

\subsection{Cell Culture. Nerve growth factor- (NGF-) differentiated} rat adrenal pheochromocytoma (PC12) cells were obtained from the Shanghai Institute of Cell Biology, Chinese Academy of Sciences. Cells were cultured in Dulbecco's modified Eagle's medium (DMEM, Gibco, NY, USA) with 10\% fetal bovine serum (FBS, PAA, Queensland, Australia), $100 \mathrm{U} / \mathrm{mL}$ penicillin, and $100 \mathrm{U} / \mathrm{mL}$ streptomycin (Ameresco, OH, USA), at $37^{\circ} \mathrm{C}$ in a humidified atmosphere of $5 \% \mathrm{CO}_{2}$ and $95 \%$ air. The growth medium was changed every other day, and cells were inoculated on 96-well plates or Petri dishes at a density appropriate to the experimental requirements.

\subsection{Oxygen-Glucose Deprivation (OGD) and Drug Treatment.} The PC12 cell cultures were subjected to OGD for $12 \mathrm{~h}$ by incubation in a deoxygenated glucose-free balanced salt solution. The cells were cultured in the DMEM medium without glucose and incubated at $37^{\circ} \mathrm{C}$ in a humidified atmosphere of $5 \% \mathrm{CO}_{2}, 94 \% \mathrm{~N}_{2}$, and $1 \% \mathrm{O}_{2}$. There were six different groups in the experiments: control, OGD exposure (model), various concentrations of YQFM $(100-400 \mu \mathrm{g} / \mathrm{mL})$, and the antioxidant Dan $(30 \mu \mathrm{M})$ as a positive control for $12 \mathrm{~h}$ during OGD treatment. YQFM was dissolved in DMEM culture medium without glucose and Dan was dissolved in DMSO (SunShineBio, Nanjing, China) at the final concentration of $0.1 \%$. 
2.6. Evaluation of Infarct Volume, Neurological Deficits, and Cerebral Blood Flow. To confirm whether YQFM exerts neuroprotective effect in vivo, the mice were randomly divided into 4 groups: Sham, Sham + YQFM, MCAO, and MCAO + YQFM. Mice were given YQFM at $1.342 \mathrm{~g} / \mathrm{kg}$ (equal to 2 times the clinical dose, according to [21]) or equal volume of $0.9 \%$ sodium chloride by intraperitoneal administration after MCAO onset. Briefly, brains were quickly removed $24 \mathrm{~h}$ after MCAO. TTC staining was performed to measure the infarct volume [23]. Neurological deficit of the experimental animals was assessed according to Longa's method as reported previously [24]. Cerebral blood flow (CBF) was measured by a laser Doppler flowmetry as described previously [25]; the images were acquired at $24 \mathrm{~h}$ after ischemia.

2.7. Cell Viability. Cell viability was measured by MTT assay as reported previously [26]. Briefly, PC12 cells were plated at a density of approximately $1 \times 10^{4} /$ well in 96 -well plates and grown in DMEM overnight. Various concentrations of YQFM $(100,200$, and $400 \mu \mathrm{g} / \mathrm{mL})$ or Dan $(30 \mu \mathrm{M})$ were added in PC12 cells and then exposed to OGD for $12 \mathrm{~h}$. The cells were sequently treated with MTT solution at the final concentration of $0.5 \mathrm{mg} / \mathrm{mL}$ in normal environment and fresh medium without YQFM for $4 \mathrm{~h}$ at $37^{\circ} \mathrm{C}$, followed by the addition of $150 \mu \mathrm{L}$ of DMSO, and OD values of the 96well plates were detected with a microplate reader (Epoch, BioTek, USA) after being shaken for $10 \mathrm{~min}$, with a detection wavelength of $570 \mathrm{~nm}$, and a reference wavelength of $650 \mathrm{~nm}$. Cell viability was expressed as a percentage of control group.

2.8. Detection of Apoptotic Cells. Hoechst 33342 dye staining was used to detect the nuclear fragmentation for evaluation of cell apoptosis according to the methods described [27]. PC12 cells were seeded and treated in 24-well plate. After the treatment, the cells were stained with Hoechst 33342 $(1 \mu \mathrm{g} / \mathrm{mL})$ for $30 \mathrm{~min}$ at $4^{\circ} \mathrm{C}$ and washed with PBS twice. Then, the cells were visualized using a fluorescence microscope. Nuclear morphological changes characteristic of apoptosis such as fragmented or condensed nuclei with strong bright blue color were observed.

Cell apoptosis was also tested with Annexin V-FITC and PI staining followed by analysis with flow cytometry, according to the instruction of the Annexin V-FITC/PI detection kit (BD Biosciences, USA). Briefly, the treated cells were washed with cold PBS and dissociated using trypsin and centrifuged at 2,000 rpm for $5 \mathrm{~min}$. Then, the cells were stained with $5 \mu \mathrm{L}$ of Annexin V-FITC and $5 \mu \mathrm{L}$ of PI resuspended in Annexin $\mathrm{V}$ binding buffer $(100 \mu \mathrm{L})$ at room temperature in the dark for $10 \mathrm{~min}$. The cells were then analyzed by flow cytometry using FL1 channel for fluorescein detection and FL3 channel for PI detection.

2.9. Measurement of Caspase-3 Activity. PC12 cells were pretreated with YQFM $(100,200$, and $400 \mu \mathrm{g} / \mathrm{mL})$ and Dan $(30 \mu \mathrm{M})$ when exposed to OGD for $12 \mathrm{~h}$. The caspase- 3 activity was detected according to the manufacturer's instructions of assay kit (Beyotime, Shanghai, China) as previously
[28]. The absorbance was detected by a microplate reader at $405 \mathrm{~nm}$. Protein levels in the supernatant were measured by Bradford method. The results were expressed as active units of caspase-3 per mg protein.

2.10. Western Blot Analysis. Western blotting analyses were performed as described previously [29]. The cells and brain tissue were lysed in the buffer supplemented with protease inhibitor and centrifuged at $12,000 \mathrm{rpm}$ for $10 \mathrm{~min}$ at $4^{\circ} \mathrm{C}$ after washing with ice-cold PBS. Equal amounts of proteins $(30 \mu \mathrm{g})$ were loaded into a $12.5 \%$ SDS-PAGE and transferred to PVDF membranes (Millipore Corporation, USA) by electrophoresis. After blocking with 5\% BSA for $1.5 \mathrm{~h}$, PVDF membranes were probed overnight at $4^{\circ} \mathrm{C}$ with primary antibodies against cleaved caspase-3 (1:800, CST, USA), Bcl-2 (1:1000, CST, USA), GRP78 (1:5000, Sigma, USA), CHOP (1:1000, CST, USA), caspase-12 (1:1000, Bioworld, USA), ATF-6 (1:500, Bioworld, USA), ATF-4 (1:500, Bioworld, USA), XBP-1 (1:1000, Bioworld, USA), eIF2 $\alpha$ (1:500, Bioworld, USA), phospho-eIF $\alpha$ ( $1: 500$, Bioworld, USA), and $\beta$-actin (1:2000, Bioworld, USA). The blots were then incubated with horseradish peroxidase- (HRP-) conjugated anti-rabbit or anti-mouse secondary antibody (dilution 1:8,000) and developed with ECL reagent. The immune-reactive bands were visualized using the ChemiDoc ${ }^{\mathrm{Tm}}$ MP System (Bio-Rad, California, USA) and the results were quantified by using the Image Lab ${ }^{\text {Tw }}$ Software (version 4.1, Bio-Rad).

2.11. Statistical Analysis. All results are expressed as the mean \pm standard deviation. Statistical analysis was carried out using Student's two-tailed $t$-test for comparison between two groups and one-way analysis of variance (ANOVA) followed by Dunnett's test when the data involved three or more groups. $P<0.05$ was considered statistically significant. All analyses were performed with GraphPad Prism Version 5.01 (GraphPad Software Inc., USA).

\section{Results}

3.1. Effects of YQFM on the Infarct Size, Neurologic Deficit Scores, and Cerebral Blood Flow (CBF) in MCAO-Injured Mice. Infarct volume, neurological deficit, and cerebral blood flow were evaluated $24 \mathrm{~h}$ after MCAO. TTC-staining image and quantitative analysis of infarct volume demonstrated that the group of YQFM at the dosage of $1.342 \mathrm{~g} / \mathrm{kg}$ had much smaller infarct size than that in the MCAO group (Figures 1(a) and 1(b)). After MCAO, the neurological deficit score of the mice in MCAO + YQFM group was significantly decreased comparing to the MCAO group, which indicated that YQFM could improve neurological deficit after $24 \mathrm{~h}$ $\mathrm{MCAO}$ in mice as described in Figure 1(c). In addition, $\mathrm{CBF}$ was measured by a laser Doppler flowmetry; the result demonstrated that administration of YQFM at the dosage of $1.342 \mathrm{~g} / \mathrm{kg}$ resulted in a significant increase on CBF $24 \mathrm{~h}$ after MCAO (Figure $1(\mathrm{~d})$ ), which indicated that the smaller infarct volume in YQFM-treated groups was correlated with improved $\mathrm{CBF}$ during $\mathrm{MCAO}$. No obvious change was 


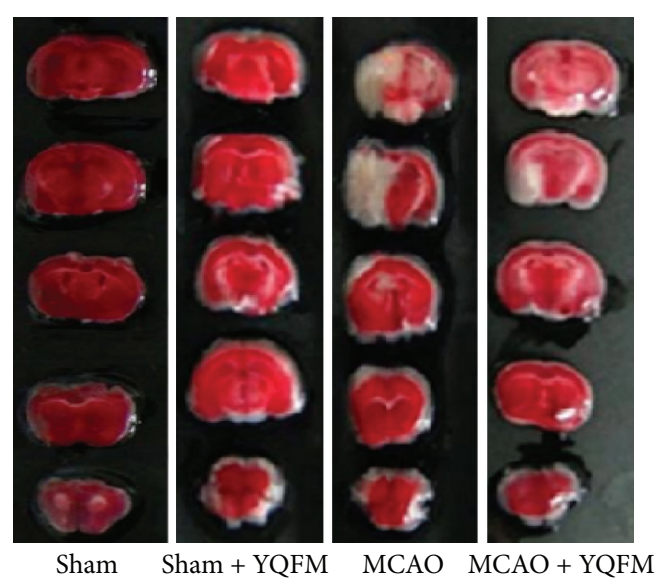

(a)

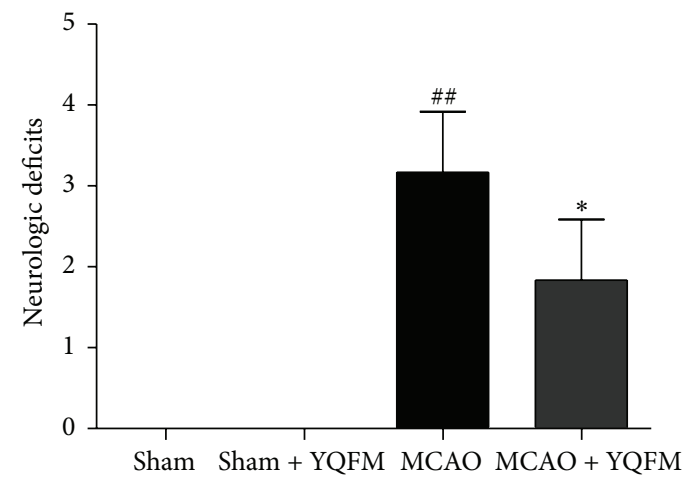

(c)

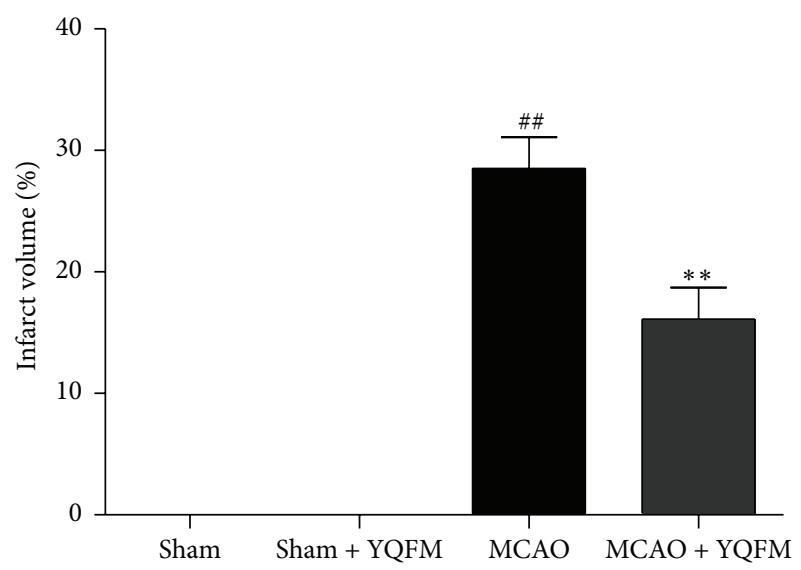

(b)

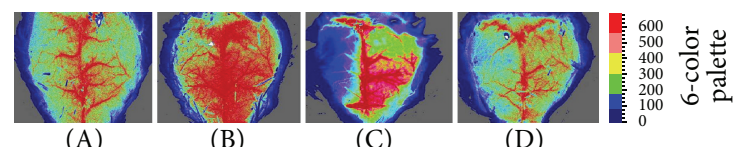

(A)

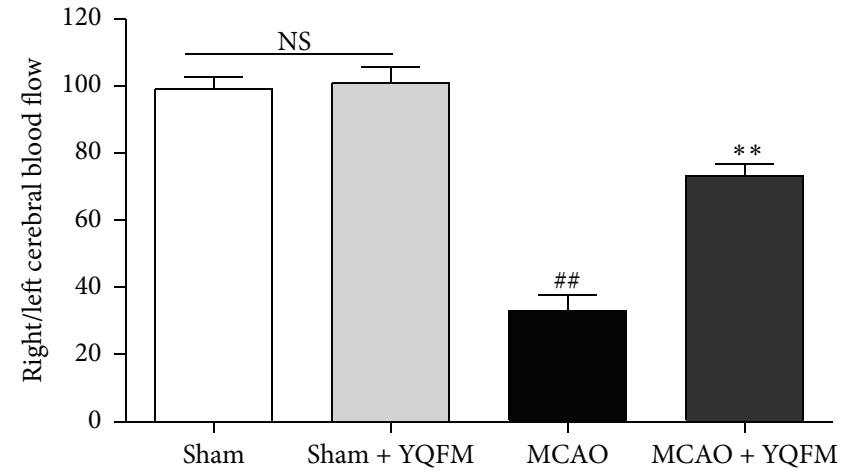

(d)

FIGURE 1: Effects of YQFM on infarct size, neurologic deficit scores and cerebral blood flow in MCAO-injured mice. Mice were subjected to $24 \mathrm{~h}$ cerebral ischemia. YQFM $(1.342 \mathrm{~g} / \mathrm{kg})$ was given by intraperitoneal administration after ischemia. (a) Representative TTC-stained brain sections in different groups. (b) Quantitative analysis of infarct volume in different groups. (c) Quantitative analysis of neurologic deficit scores in different groups. (d) The magnitude of CBF is represented by different colors and quantitative analysis of CBF in different groups. Data were presented as mean $\pm \mathrm{SD} .{ }^{\# \#} P<0.01$ versus Sham, ${ }^{*} P<0.05,{ }^{* *} P<0.01$ versus MCAO $(n=6 \sim 8)$.

observed between Sham and Sham + YQFM groups in above experiments (Figure 1).

\subsection{Effect of YQFM on ER Stress-Related Proteins in MCAO-} Injured Mice. The expressions of ER stress-related marker proteins and signaling pathways were examined with Western blot. As demonstrated in Figure 2, the administration of YQFM $(1.342 \mathrm{~g} / \mathrm{kg})$ notably increased the expression of Bcl2 and decreased the expressions of caspase-12, GRP78, and CHOP. All these data indicate that YQFM improves cerebral ischemic injury linked with ER stress in MCAO mice. No obvious change was observed between Sham and Sham + YQFM groups in above experiments (Figure 2).

3.3. Effect of YQFM on ER Stress-Related Signaling Pathways in MCAO-Injured Mice. The expressions of ER stress-related signaling pathways were examined with Western blot. The Western blot results demonstrated that YQFM treatment could inhibit the expressions of ATF-4, ATF-6, XBP-1, and phospho-eIF $2 \alpha$ in MCAO mice. No obvious change was observed between Sham and Sham + YQFM groups in those results. All these data indicate that YQFM improves cerebral ischemic injury linked with ER stress-related signaling pathways in MCAO mice (Figure 3).

3.4. Effect of YQFM on OGD-Injured Cell Viability in PC12 Cells. We investigated the injurious effects of different time of OGD on PC12 cells using MTT assay. The result demonstrated that the cell viability significantly decreased by approximately $40 \%$ when the cultured PC12 cells were exposed to OGD for $12 \mathrm{~h}$ (Figure 4(a)). Therefore, we adopted OGD for $12 \mathrm{~h}$ in the subsequent experiments. The effect of 


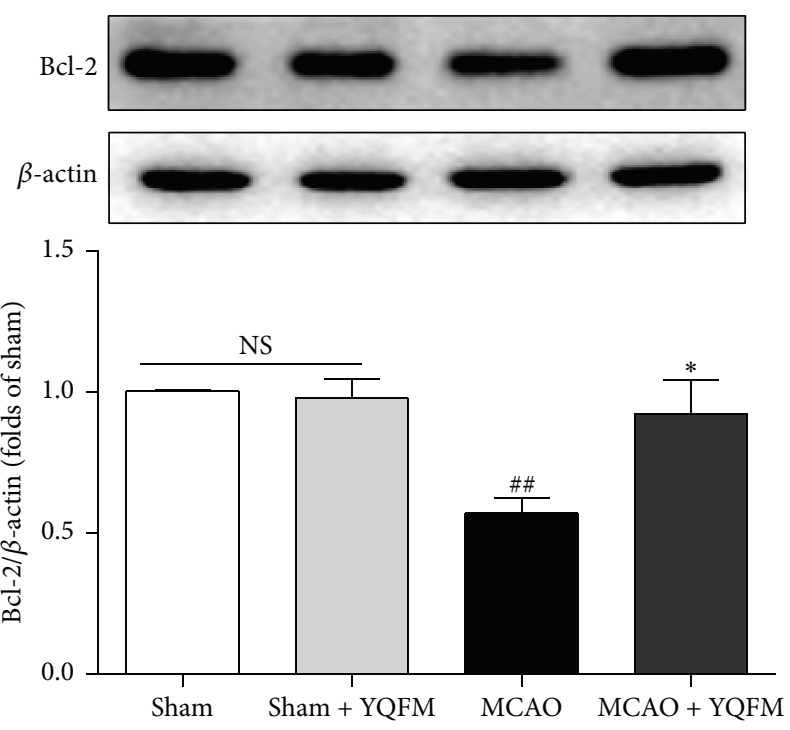

(a)
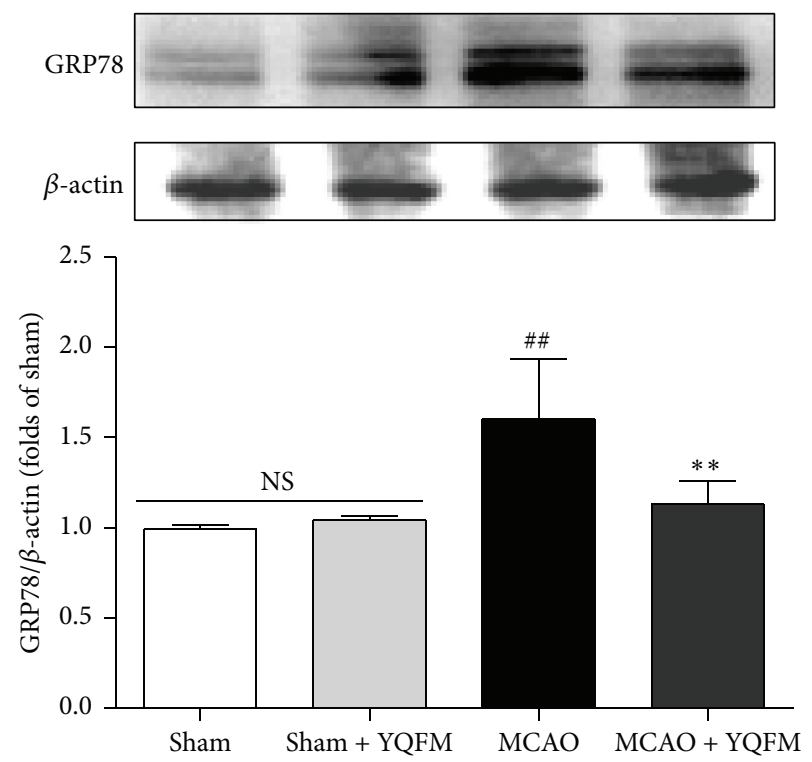

(c)

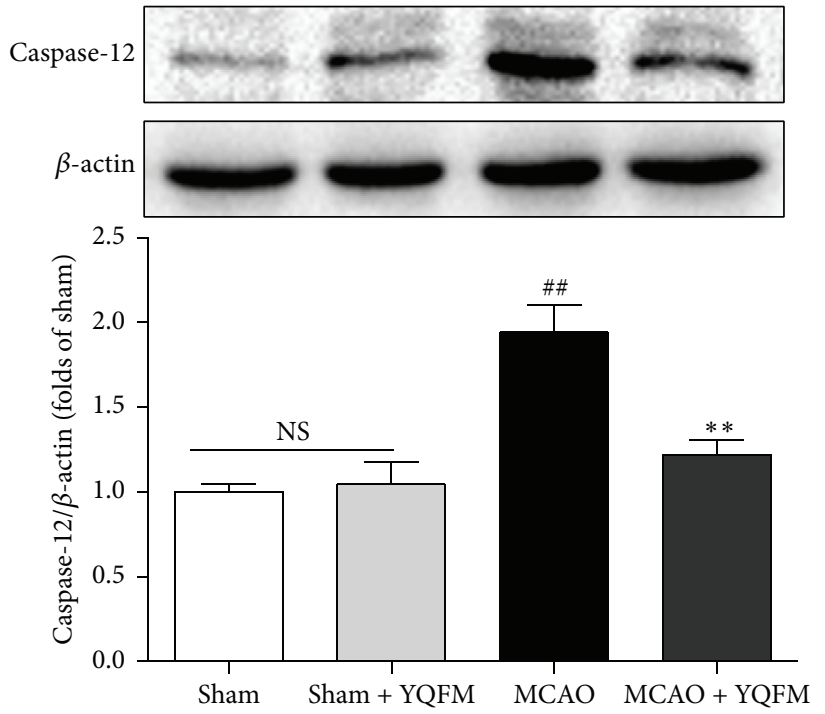

(b)
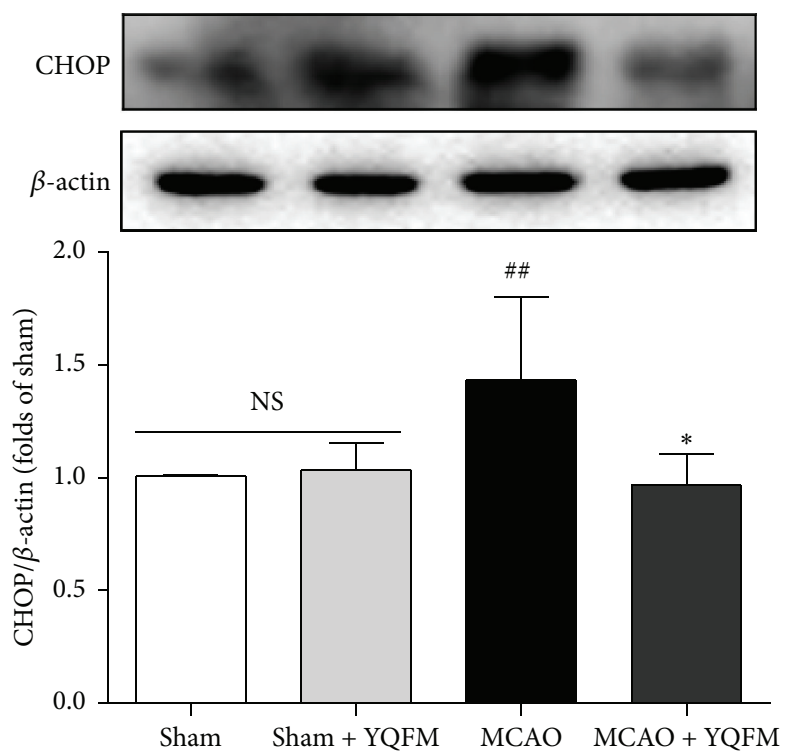

(d)

FIGURE 2: Effects of YQFM on ER stress-related proteins in MCAO-injured mice. Mice were subjected to $24 \mathrm{~h}$ of cerebral ischemia, and YQFM $(1.342 \mathrm{~g} / \mathrm{kg})$ was given by intraperitoneal administration after ischemia. Representative Western blot and the quantitative analysis of the ratio of (a) Bcl-2, (b) caspase-12, (c) GRP78 and (d) CHOP. Data were presented as mean \pm SD. ${ }^{\# \#} P<0.01$ versus Sham, ${ }^{*} P<0.05$, and ${ }^{* *} P<0.01$ versus $\mathrm{MCAO}(n=3)$.

various concentrations of YQFM $(100-400 \mu \mathrm{g} / \mathrm{mL})$ pretreatment on OGD-injured ( $12 \mathrm{~h}$ explosion) PC12 cells was also investigated with Dan at the dosage of $30 \mu \mathrm{M}$ as the positive control. The result demonstrated that the OGD-induced reduction of PC12 cell viability was significantly increased by the pretreatment of YQFM in various concentrations of 100$400 \mu \mathrm{g} / \mathrm{mL}$ or Dan $(30 \mu \mathrm{M})$ (Figure $4(\mathrm{~b}))$.

3.5. Effect of YQFM on OGD-Induced Apoptosis in PC12 Cells. Whether YQFM could protect against neuronal injury via the inhibition of apoptosis needs to be confirmed. As the Hoechst 33342 staining result demonstrated, the fluorescence intensity was decreased with the presence of the different concentrations of YQFM $(100-400 \mu \mathrm{g} / \mathrm{mL})$ and Dan $(30 \mu \mathrm{M})$, comparing to the MCAO model group (Figure 5(a)). Correspondingly, when exposed to OGD injury, the activity of caspase-3, final executor of apoptosis, was remarkably elevated, comparing to the control group, while the pretreatment of YQFM $(100-400 \mu \mathrm{g} / \mathrm{mL})$ or Dan $(30 \mu \mathrm{M})$ significantly decreased the activity of caspase-3 (Figure 5(b)). In addition, 


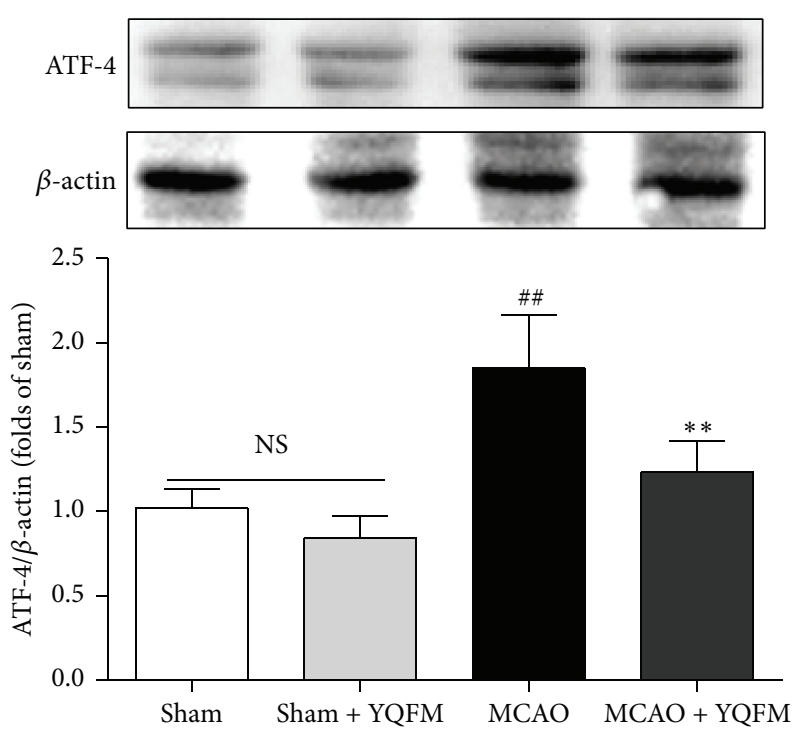

(a)
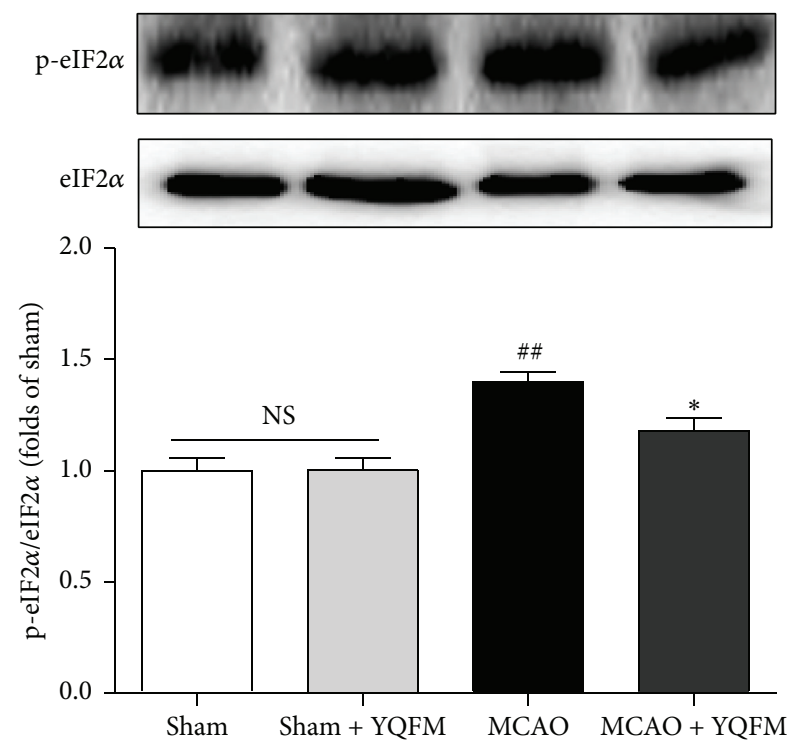

(c)
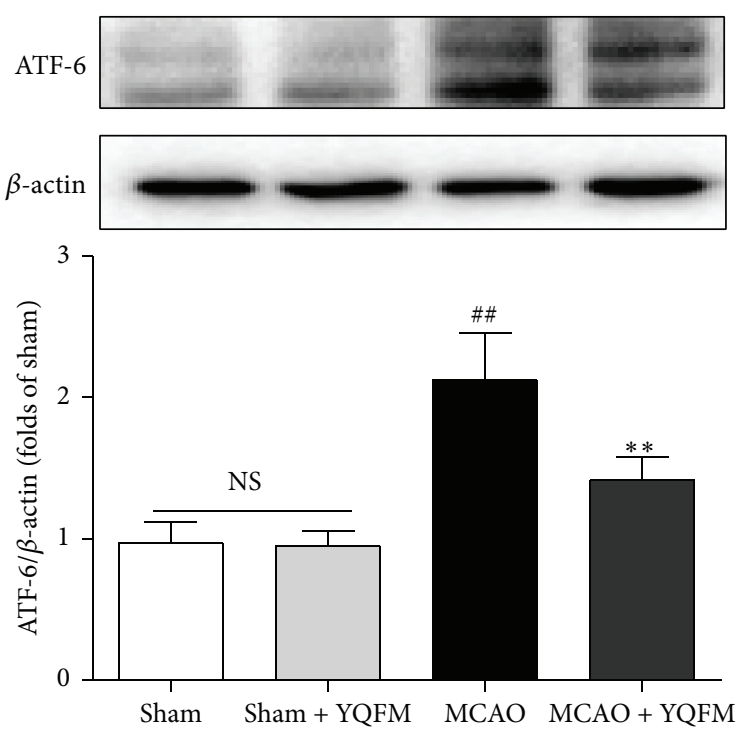

(b)
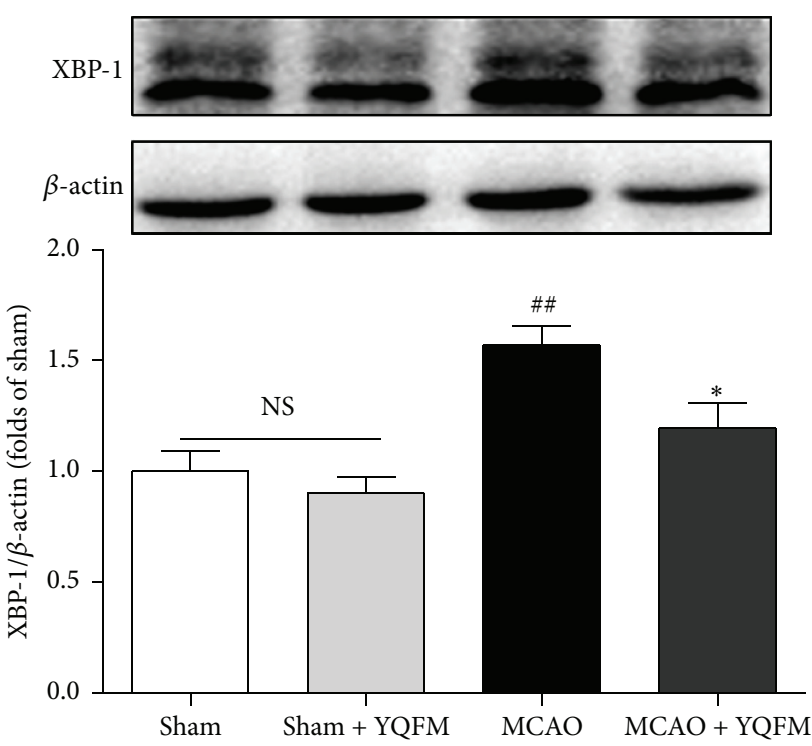

(d)

FIGURE 3: Effects of YQFM on ER stress-related signaling pathways in MCAO-injured mice. Mice were subjected to $24 \mathrm{~h}$ of cerebral ischemia, and YQFM $(1.342 \mathrm{~g} / \mathrm{kg})$ was given by intraperitoneal administration after ischemia. Representative Western blot and the quantitative analysis of the ratio of (a) ATF- 4 and (b) ATF- 6 and (c) the ratio of p-eIF2 $\alpha /$ IF $2 \alpha$ and (d) XBP-1. Data were presented as mean \pm SD. ${ }^{\# \#} P<0.01$ versus Sham, ${ }^{*} P<0.05$, and ${ }^{* *} P<0.01$ versus $\mathrm{MCAO}(n=3)$.

Annexin V-FITC/PI was used to detect early apoptotic cells and identify late-stage apoptosis due to its ability to penetrate into the nuclear of apoptotic cells, while it is the opposite in normal cells (from $9.37 \%$ to $33.13 \%$ ). The flow cytometry results demonstrated that the pretreatment of YQFM at the three concentrations or Dan $(30 \mu \mathrm{M})$ could significantly rescue the PC12 cells exposed for $12 \mathrm{~h}$ from apoptosis with the percentage of $24.73 \%, 17.53 \%, 14.06 \%$, and $18.13 \%$, respectively. (Figures 5(c) and 5(d)).

Furthermore, we investigated the effects of YQFM on the expression of apoptosis-related proteins, such as cleaved caspase-3 and Bcl-2 in PC12 cells. According to Western blot, the treatment with YQFM or Dan decreased the cleaved caspase- 3 expression and increased Bcl-2 expression, comparing to the OGD model group (Figures 5(e) and 5(f)).

3.6. Effect of YQFM on the Expression of ER Stress-Related Proteins in OGD-Induced PC12 Cells. To investigate the involvement of ER stress in the neuroprotective effect of YQFM, we measured the ER apoptosis specific activator protein, cleaved caspase-12, and the expressions of ER stress markers, CHOP 


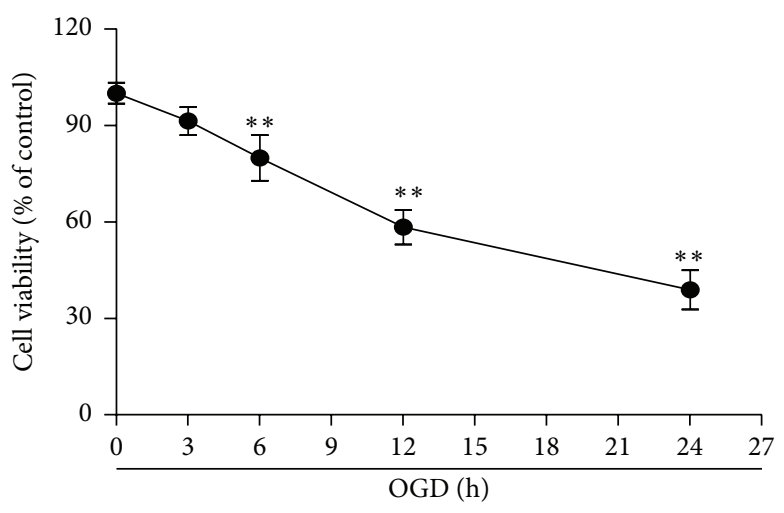

(a)

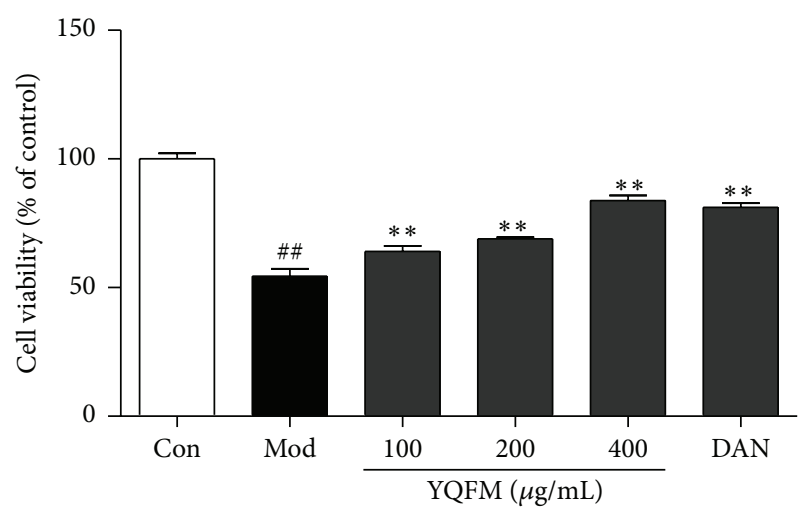

(b)

FIGURE 4: Effect of YQFM on the OGD-injured cell viability in PC12 cells. (a) Cell viability was measured by MTT assay after OGD for 3, 6, $12,18,21,24$, and $27 \mathrm{~h}$. (b) PC12 cells were treated with YQFM at the concentrations of 100, 200, and $400 \mu \mathrm{g} / \mathrm{mL}$ for $12 \mathrm{~h}$ during OGD. Cell viability was measured by MTT assay. Data were presented as mean $\pm \mathrm{SD} .{ }^{\# \#} P<0.01$ versus control, ${ }^{*} P<0.05$, and ${ }^{* *} P<0.01$ versus model $(n=6)$.

and GRP78, with Western blot. The results demonstrated that the pretreatment of YQFM at 200 and $400 \mu \mathrm{g} / \mathrm{mL}$ or Dan $(30 \mu \mathrm{M})$ could inhibit the increased expression of caspase12, CHOP, and GRP78 in OGD-induced PC12 cells. The percentages of inhibition were $74.35 \%, 94.66 \%$, and $99.81 \%$, respectively (YQFM $(400 \mu \mathrm{g} / \mathrm{mL})$ versus model) (Figure 6).

\subsection{Effect of YQFM on ER Stress-Related Signaling Pathways} in OGD-Induced PC12 Cells. To explore the underlying mechanism of the inhibition effect of YQFM on ES stressrelated neuronal apoptosis, we investigated the effects of YQFM on the UPR-associated genes, such as ATF-4, ATF6 , eIF $2 \alpha$, phospho-eIF $2 \alpha$, and XBP-1 in PC12 cells. The Western blot results demonstrated that the expressions of ATF-4, ATF-6, XBP-1, and phosphor-eIF2 $\alpha$ were upregulated when exposed to OGD for $12 \mathrm{~h}$, while the treatment of YQFM at three concentrations significantly downregulated the triggered protein expressions, corresponding to that of the positive drug, Dan $(30 \mu \mathrm{M})$. The percentage of inhibition was $62.67 \%, 89.46 \%, 68.97 \%$, and $81.04 \%$, respectively (YQFM $(400 \mu \mathrm{g} / \mathrm{mL})$ versus model) (Figure 7).

\section{Discussion}

In the present study, we confirmed the neuroprotective effect of YQFM and explored the role of ER stress-mediated neuronal apoptosis in MCAO-injured mice and OGD-injured PC12 cells. In vivo results demonstrated that the treatment of YQFM $(1.342 \mathrm{~g} / \mathrm{kg})$ could reduce brain infarct size, improve neurological deficits and increase $\mathrm{CBF}$, and regulate the expression of ER stress-related proteins and signaling pathways in MCAO mice brain. The in vitro results correspondingly indicated that YQFM $(100,200$, and $400 \mu \mathrm{g} / \mathrm{mL})$ could increase the injured cell viability, inhibit cell apoptosis, and regulate the ER stress-related proteins and signaling pathways in OGD-induced PC12 cells.
Series of medicines, such as dantrolene and edaravone, which have been proved to be effective in attenuating ER stress after experimental cerebral ischemia, may become a potent therapeutic agent as the treatment for ischemic brain disease in the near future $[5,15,30-32]$. Dantrolene, used as a ryanodine receptor antagonist, can significantly decrease infarct volume and provide neuroprotective effect on rats after $1.5 \mathrm{~h}$ of MCAO and $24 \mathrm{~h}$ of reperfusion via reducing ER stress-mediated apoptotic signaling pathways by inhibiting the expressions of eIF $2 \alpha$ phosphorylation, ATF- 4 , and $\mathrm{CHOP}$ [30]. Thus, dantrolene was used as the positive control in this present study and we confirmed that YQFM indicated similar effect of dantrolene in the ischemic stroke model mice. Moreover, our data further indicated that YQFM could inhibit the expressions of GRP78, caspase-12, ATF-6, and XBP-1 in MCAO-injured mice for $24 \mathrm{~h}$.

ER stress is an essential step in the progression of MCAO injury and plays an important role in ischemic neuronal cell apoptosis as previously reported [16, 33]. Remote ischemic postconditioning can decrease the protein level of phosphoeIF2 $\alpha$, caspase-12, and CHOP, which protects against brain injury in rats of MCAO and reperfusion by attenuating ER stress response-induced apoptosis [34]. It is reported that nafamostat mesilate (NM), a type of serine protease inhibitor, can attenuate MCAO and reperfusion-induced brain injury via the inhibition of GRP78, CHOP, and phospho-eIF2 $\alpha$ [35]. Therefore, modulation on ER stress exerts a remarkable protective effect on the ischemic brain and provides the prospect of new stroke therapies. Here, we verified the protective effect of YQFM and its possible mechanism related with ER stress in vivo. According to our data, YQFM could reduce brain infarct size, improve neurological deficits, and increase CBF in $\mathrm{MCAO}$-injured mice, which further confirmed the protective effect of YQFM in ischemic stroke, corresponding to the previous study [18]. Moreover, YQFM also could regulate the expressions of ER stress-related proteins and signaling 


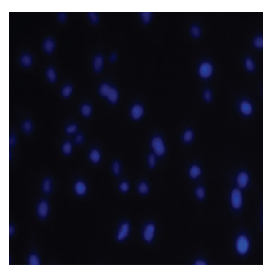

(A)

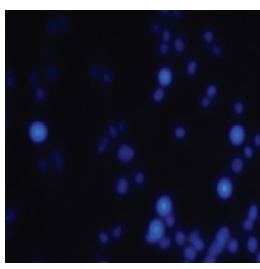

(D)

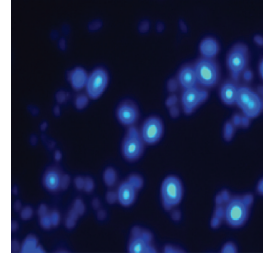

(B)

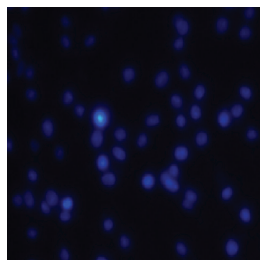

(E)

(a)

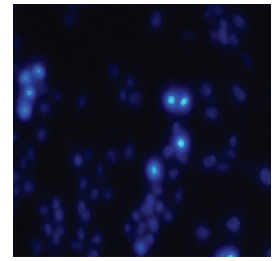

(C)

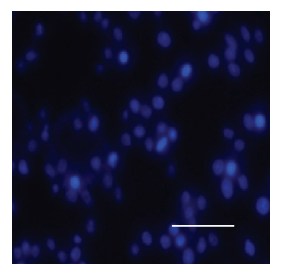

(F)

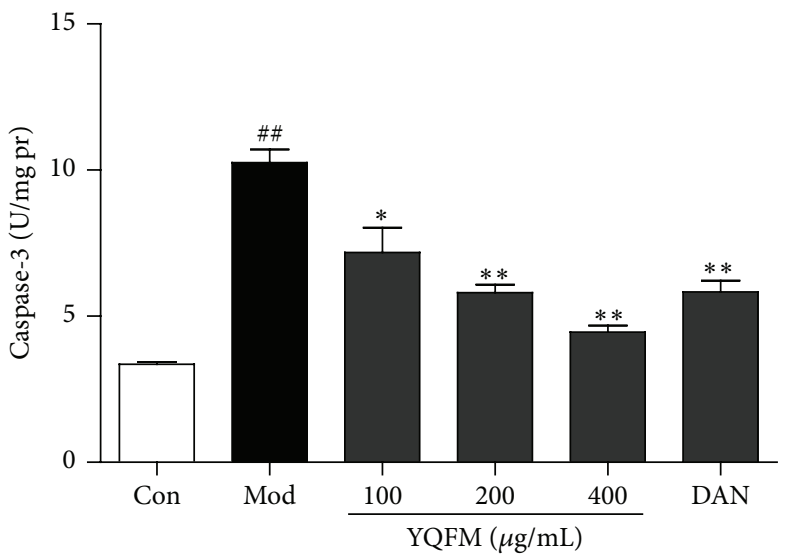

(b)

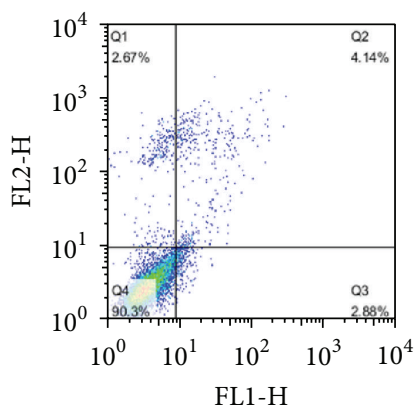

(A)

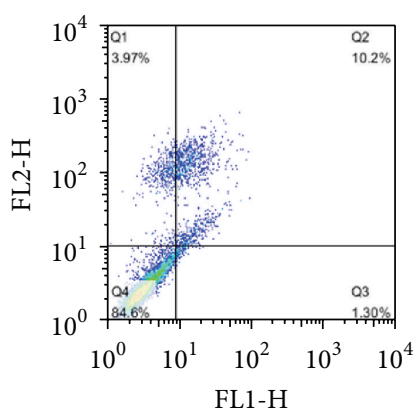

(D)

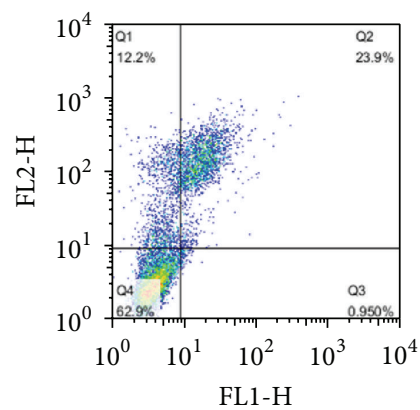

(B)

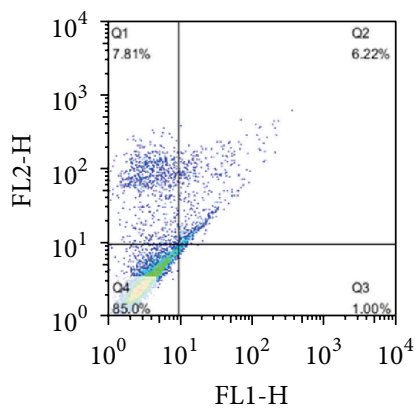

(E)

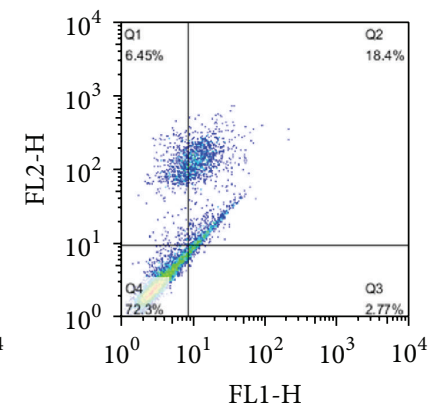

(C)

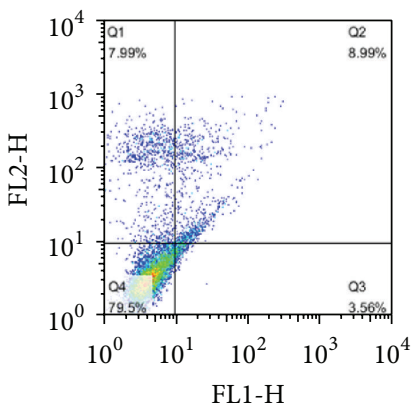

(F)

(c)

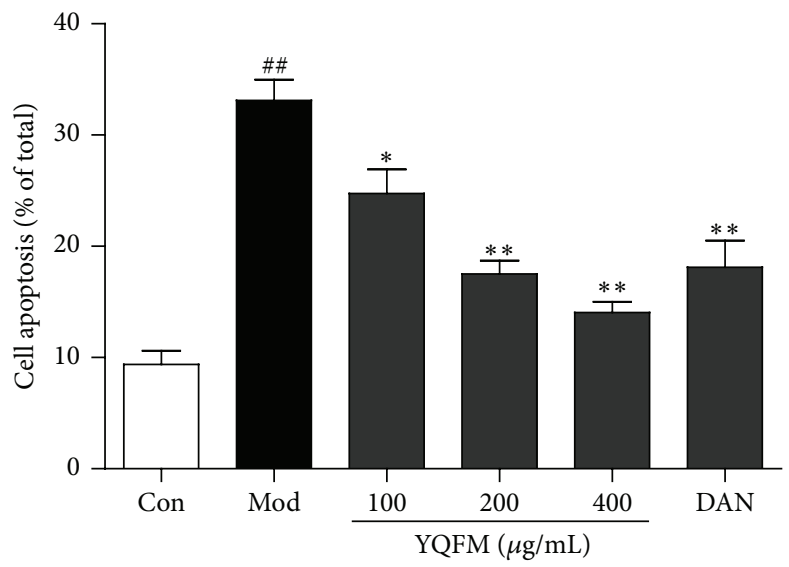

(d)

Figure 5: Continued. 


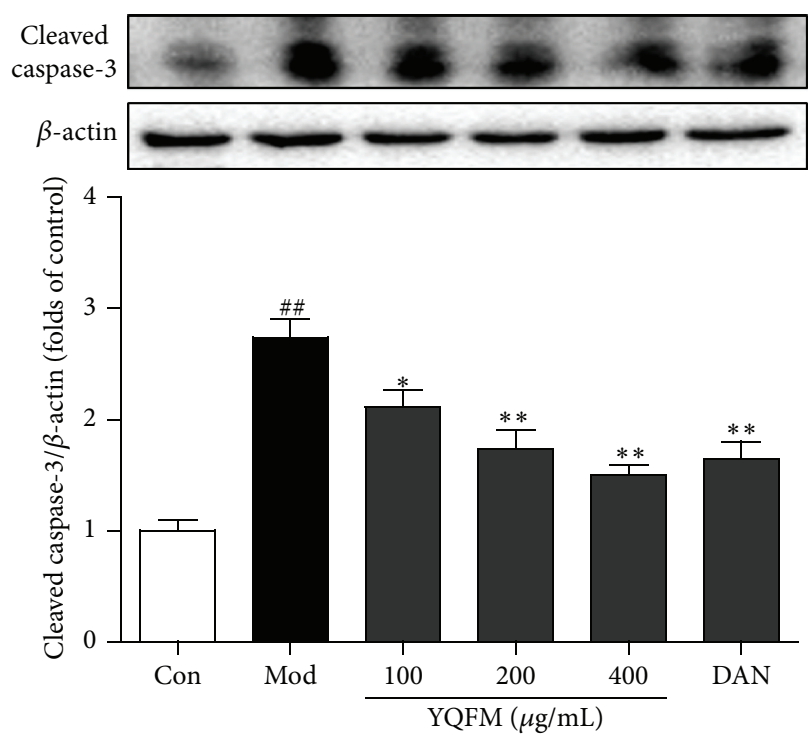

(e)

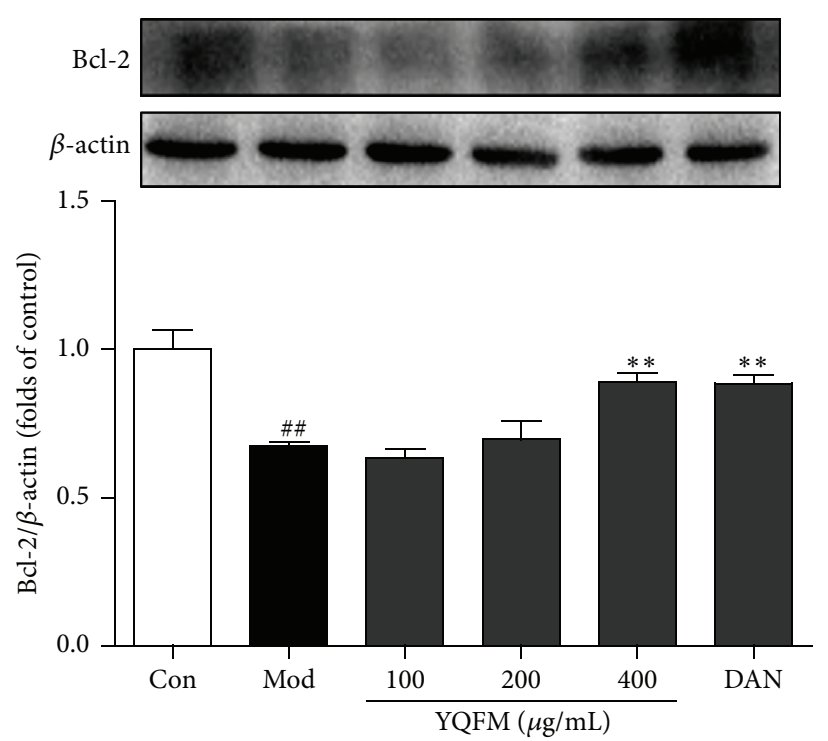

(f)

Figure 5: Effect of YQFM on OGD-induced cell apoptosis in PC12 cells. (a) PC12 cells were treated with YQFM (100, 200, and 400 $\mu \mathrm{g} / \mathrm{mL})$ or Dan $(30 \mu \mathrm{M})$ for $12 \mathrm{~h}$ during OGD before Hoechst 33342 staining (200x, final magnification) as follows: control group; model group; group pretreated with YQFM at the concentrations of 100,200 , and $400 \mu \mathrm{g} / \mathrm{mL}$ and then exposed to OGD; group pretreated with $30 \mu \mathrm{M}$ Dan and then exposed to OGD. Scale bar $=50 \mu \mathrm{m}$. (b) Quantitative analysis of caspase-3 activity which was tested by test kit. (c) Cell apoptosis was tested by flow cytometry analysis as follows: control group; model group; group pretreated with YQFM at the concentrations of 100, 200, and $400 \mu \mathrm{g} / \mathrm{mL}$ and then exposed to OGD; group pretreated with $30 \mu \mathrm{M}$ Dan and then exposed to OGD. (d) The rate of cell apoptosis (\%) was analyzed by flow cytometry analysis. The expressions of (e) cleaved caspase-3 and (f) Bcl-2 were detected by Western blot. Blots normalized to $\beta$-actin expression were shown. Data were presented as mean $\pm \mathrm{SD}$. ${ }^{\# \#} P<0.01$ versus control, ${ }^{*} P<0.05$, and ${ }^{* *} P<0.01$ versus model $(n=3)$.

pathways, modulating the expressions of not only GRP78, caspase-12, CHOP, and phospho-eIF $2 \alpha$, but also ATF- 4 , ATF6 , and XBP-1 (Figures 2 and 3). The results suggested that YQFM could regulate some other ER stress-related pathways in MCAO-injured mice. All these findings demonstrate YQFM can improve MCAO-induced brain damage in mice, and the beneficial effects may be linked with ER stress-related signaling pathways.

In addition, the previous studies have demonstrated that OGD exposure can result in cell viability reduction and cell apoptosis in many different cell types, including neuronal cells, astrocyte, and vascular endothelial cells [3638]. Furthermore, NGF-differentiated PC12 cells have been extensively used as a neuronal cells line model for the nervous system study, and with regard to the in vitro study of hypoxia, an OGD model of PC12 cells has been established [39, 40]. Therefore, we evaluated the efficacy of YQFM in OGDinduced PC12 cells in this present study. We found that incubation with YQFM increased cell viability and decreased the ratio of cell apoptosis by the MTT assay and Hoechst 33342 staining and Annexin V-FITC/PI analysis (Figures 4, 5(a), and 5(b)). Moreover, YQFM could inhibit the expression of cleaved caspase- 3 and increase the expression of $\mathrm{Bcl}$ 2 (Figures 5(e) and 5(f)), which are the most important apoptosis-related proteins in the process of cell apoptosis [41].
Therefore, our findings indicate YQFM can protect PC12 cells from OGD-induced injury via inhibiting cell apoptosis.

ER stress is a major intracellular signal transduction pathway of apoptosis [11, 33, 42]. Several studies have suggested that ER stress plays a critical role in a variety of processes including ischemia and hypoxia $[8,34,43]$. Moreover, induction of ER stress in response to OGD also involves the activation of the protein kinase RNA- (PKR-) like ER kinase- (PERK-) eIF2 $\alpha$-ATF4 and IRE1-XBP1 pathways, and the increased expression of caspase-12, GRP78, and CHOP $[8,11,44]$. Thus, we then pointed to whether attenuation of ER stress-induced apoptotic signaling pathways contributed to the mechanisms in OGD-injured PC12 cells. Our study is in good agreement with these studies that OGD could stimulate ER stress to induce apoptosis in PC12 cells, while YQFM could inhibit the expression of ER stressrelated proteins caspase-12, GRP78, and CHOP (Figure 6) and UPR-associated genes ATF-4, ATF-6, phospho-eIF $2 \alpha$, and XBP-1 (Figure 7). We also verified that YQFM could modulate the expressions of cleaved caspase- 3 and $\mathrm{Bcl}-2$, which was the direct evidence of attenuation of ER stressmediated apoptotic signaling pathways in OGD-injured PC12 cells. All these findings indicated that YQFM ameliorates OGD-induced neuronal apoptosis linked with modulating ER stress-related signaling pathways. 


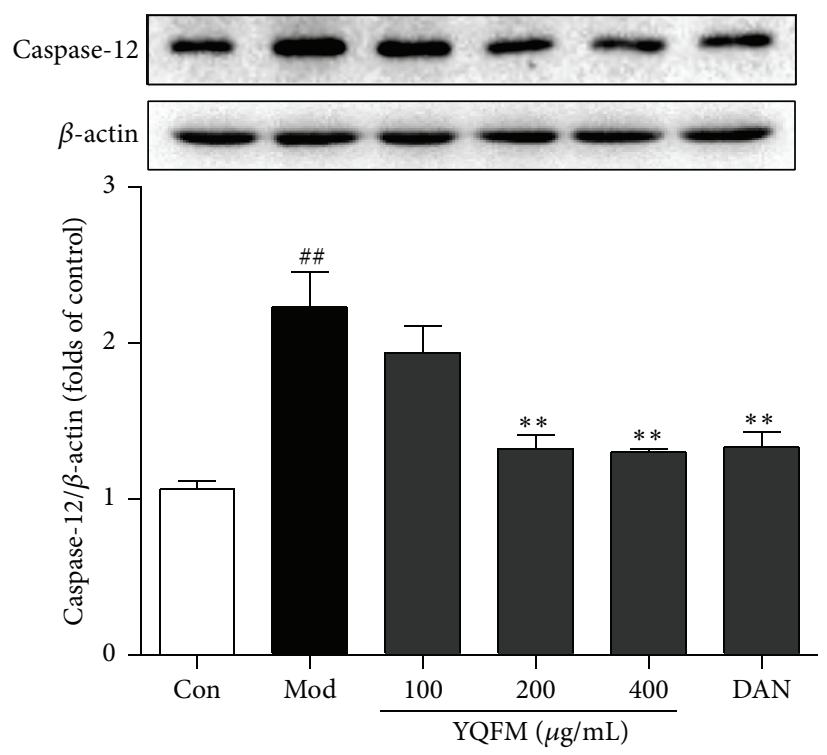

(a)
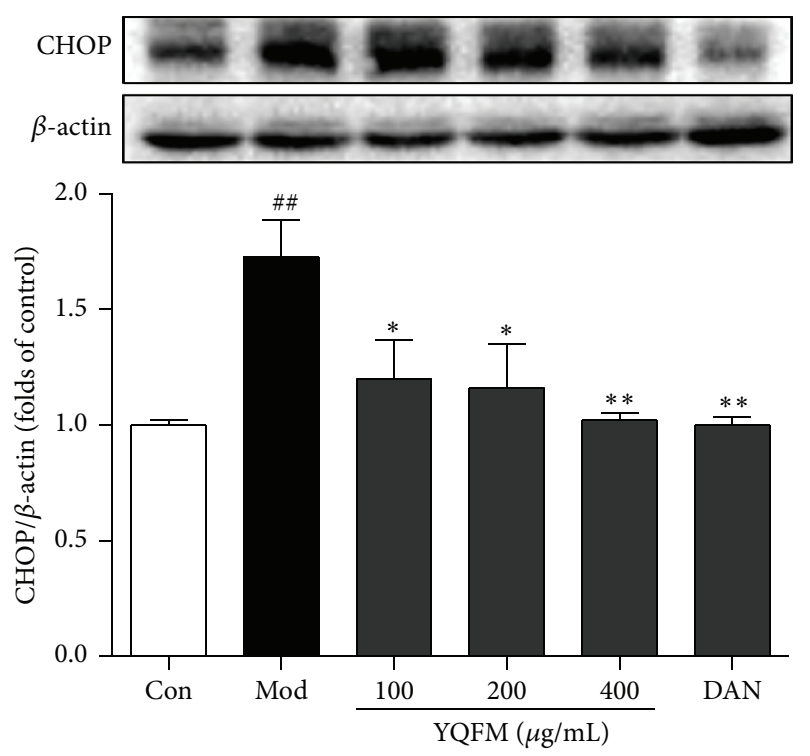

(b)

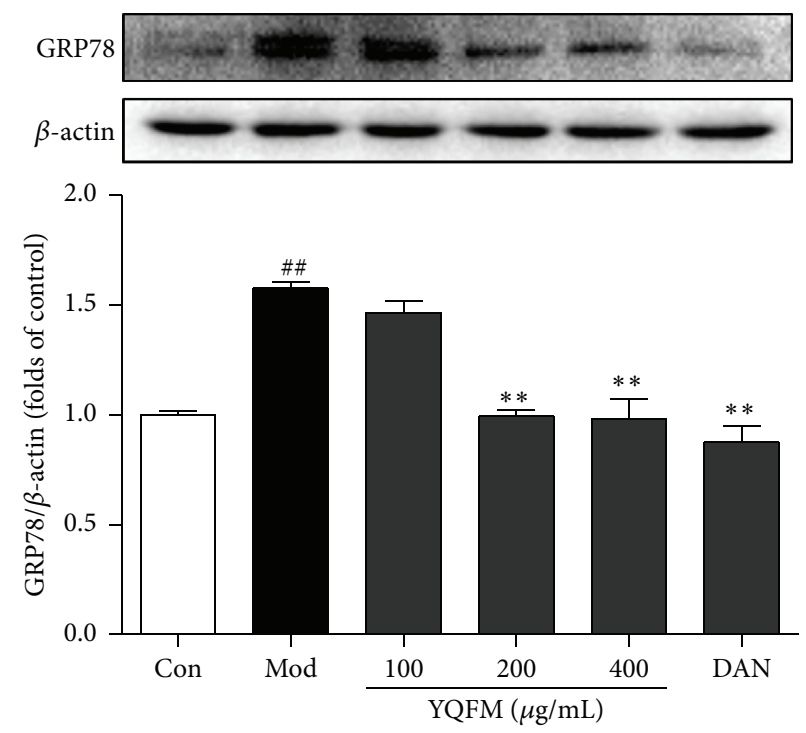

(c)

FIGURE 6: Effects of YQFM on the expression of ER stress-related proteins in OGD-induced PC12 cells. PC12 cells were treated with YQFM $(100,200$, and $400 \mu \mathrm{g} / \mathrm{mL})$ or Dan $(30 \mu \mathrm{M})$ for $12 \mathrm{~h}$ during OGD, the treated cells were lysed, and proteins were isolated and detected by Western blotting. Quantitative analysis of (a) caspase-12, (b) CHOP, and (c) GRP78 was performed by Image Lab ${ }^{\mathrm{Tx}}$ Software. Blots normalized to $\beta$-actin expression were shown. Data were presented as mean $\pm \mathrm{SD} .{ }^{\# \#} P<0.01$ versus control, ${ }^{*} P<0.05$, and ${ }^{* *} P<0.01$ versus model $(n=3)$.

Apart from these findings, the present study still has several limitations due to many other possible mechanisms existing in the ischemia-induced apoptosis model, such as extrinsic apoptosis pathway and mitochondrial apoptosis pathway [41, 45, 46]. Furthermore, whether or not YQFM can modulate cell apoptosis through other related signaling pathways still remains unclear. In the current study, we evaluated the effects of YQFM on GRP78-XBP1-ATF6eIF2 $\alpha$ signaling pathway in vitro. However, other signaling pathways related to ER stress need to be further examined. In addition, as previously described [47], YQFM consists of various bioactive compounds, such as ginsenoside Rg1, ginsenoside Rbl, and schisandrin, some of which display neuroprotective effects after OGD injury [26, 48]. Moreover, some reports have demonstrated that those bioactive compounds can inhibit cell apoptosis by inducing ER stress. For example, ginsenoside Rg1 can exhibit neuroprotective effects by inhibiting the ER stress-mediated c-Jun N-terminal 


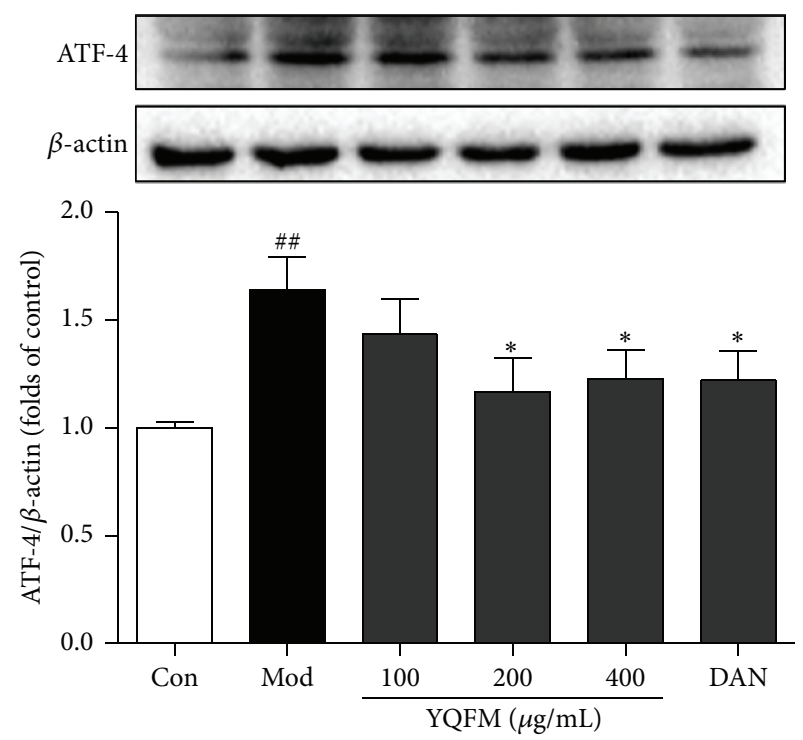

(a)
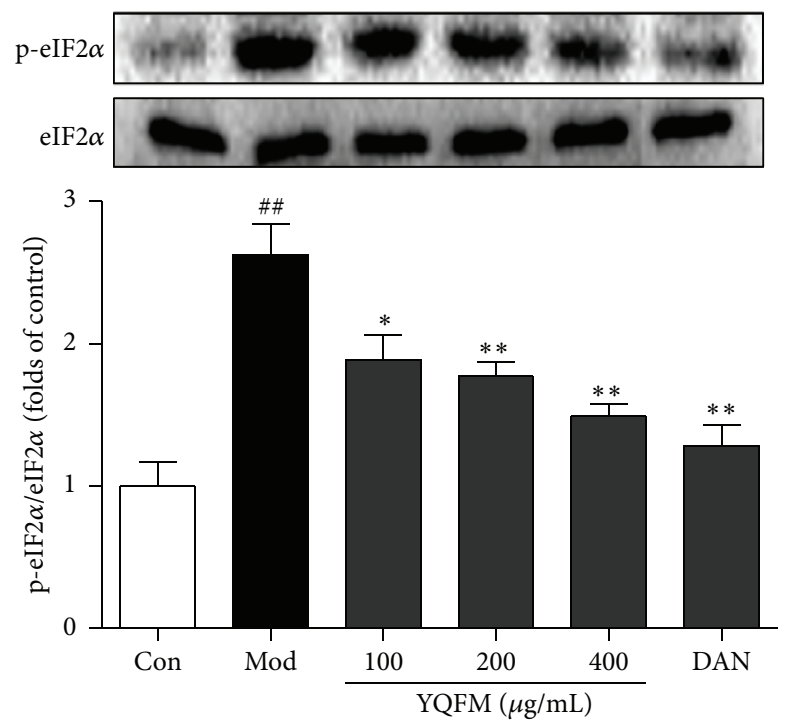

(c)

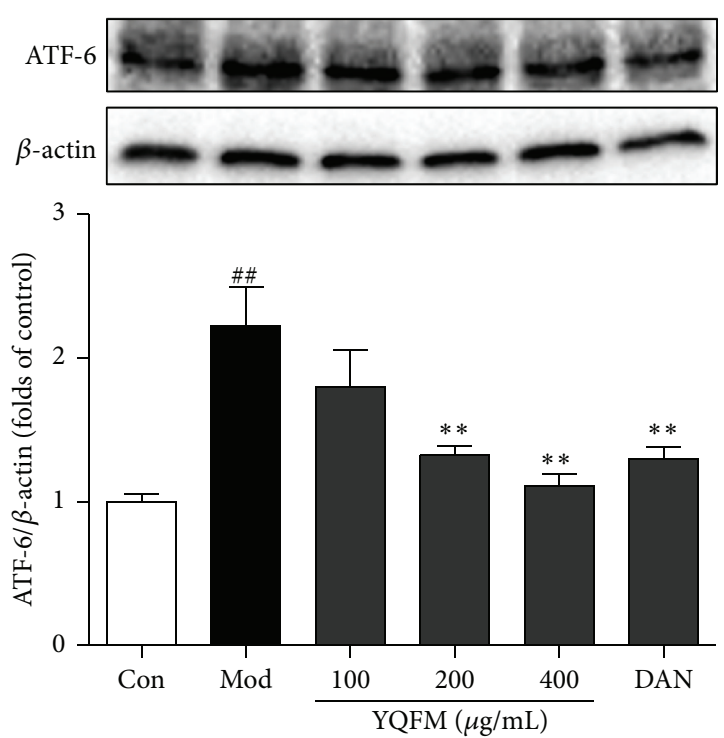

(b)

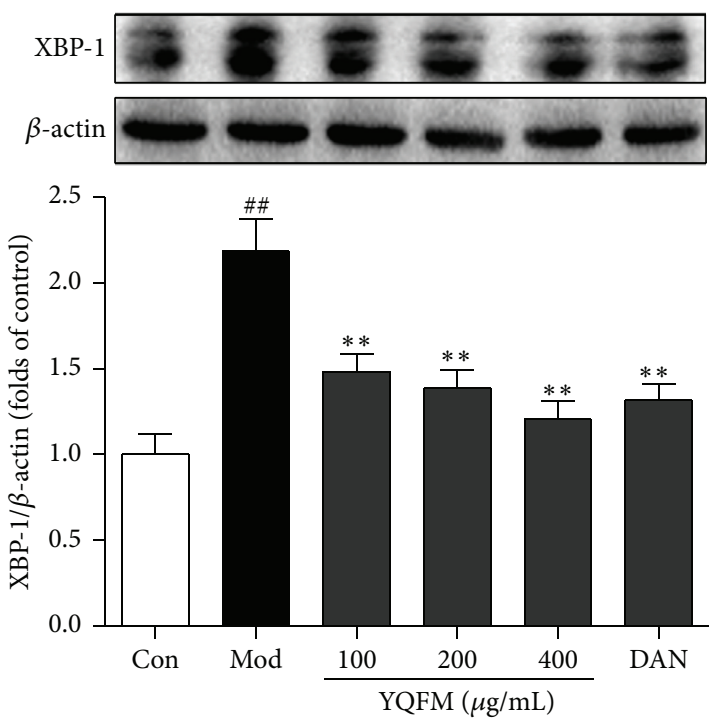

(d)

FIGURE 7: Effects of YQFM on ER stress-related signaling pathways in OGD-induced PC12 cells. PC12 cells were treated with YQFM (100, 200 , and $400 \mu \mathrm{g} / \mathrm{mL}$ ) or Dan $(30 \mu \mathrm{M})$ for $12 \mathrm{~h}$ during OGD, and then cells were lysed and proteins were isolated. The expressions of (a) ATF-4 and (b) ATF-6 and (c) the ratio of p-eIF2 $\alpha /$ IF $2 \alpha$ and (d) XBP-1 were detected by Western blotting. Blots normalized to $\beta$-actin expression were shown. Data were presented as mean \pm SD. ${ }^{\# \#} P<0.01$ versus control, ${ }^{*} P<0.05$, and ${ }^{* *} P<0.01$ versus model $(n=3)$.

kinase (JNK) apoptotic pathway in a rat model of Alzheimer's disease, and ginsenoside Rbl also can suppress the activation of ER stress-associated proteins including PERK and CHOP, and downregulation of Bcl-2 induced by high glucose [49, 50]. These findings prompted that YQFM could inhibit ER stress-mediated neuronal apoptosis linked with JNK and PERK apoptotic signaling pathways. Therefore, how those bioactive compounds or their combination contributes to the global activities of YQFM needs further investigation. On the other hand, ER stress and oxidative stress are linked to multiple human pathologies, including metabolic, neurodegenerative, immune/inflammatory, and neoplastic diseases [51-53], and whether YQFM could be beneficial for these diseases is of a great interest to explore.

In conclusion, our findings demonstrate that YQFM treatment provides a protective effect against brain damage induced by ischemia injury in vivo and in vitro, and the attenuation of ER stress-mediated neuronal apoptosis may be involved in the mechanisms of YQFM (schematic graph is shown in Figure 8). Our findings provide the experimental 


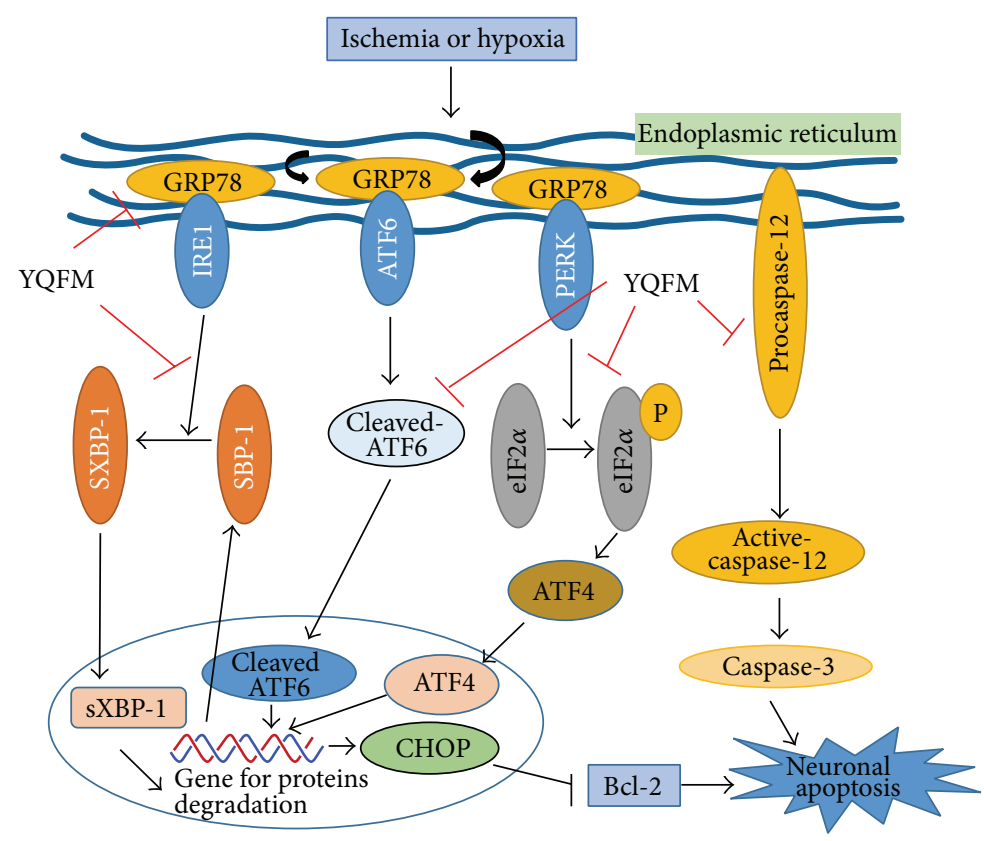

FIGURE 8: Proposed mechanisms of YQFM on ER stress-mediated neuronal apoptosis in cerebral ischemia injury. Under conditions of physiological homeostasis, PERK, ATF-6, and IRE1 interact with GRP78 in ER. Upon hypoxia or ischemia, upregulated GRP78 activates the autophosphorylation of IRE-1 and PERK and triggers XBP-1, ATF-6, and eIF $2 \alpha$ phosphorylation. Phosphorylation of eIF2 $\alpha$ activates the ATF-4. All those three pathways contribute to gene for protein degradation and activate CHOP, which inhibits the antiapoptotic protein, Bcl2. Moreover, activated caspase-12 activates caspase-3, leading to enhanced neuronal apoptosis and cerebral damage. YQFM inhibits neuronal apoptosis and protects brain damage via modulating ER stress-related signaling pathways.

basis of YQFM for the prevention and treatment of cerebral ischemia disease, enriching the function of YQFM in clinical application.

\section{Conflict of Interests}

The authors have no conflict of interests to declare.

\section{Acknowledgments}

The present research was supported by funding from the National Natural Science Foundation of China (no. 81274004), 2011 Program for Excellent Scientific and Technological Innovation Team of Jiangsu Higher Education, a Project Funded by the Priority Academic Program Development of Jiangsu Higher Education Institutions.

\section{References}

[1] M. A. Moskowitz, E. H. Lo, and C. Iadecola, "The science of stroke: mechanisms in search of treatments," Neuron, vol. 67, no. 2, pp. 181-198, 2010.

[2] K. P. Doyle, R. P. Simon, and M. P. Stenzel-Poore, "Mechanisms of ischemic brain damage," Neuropharmacology, vol. 55, no. 3, pp. 310-318, 2008.

[3] G. A. Donnan, M. Fisher, M. Macleod, and S. M. Davis, "Stroke," The Lancet, vol. 371, no. 9624, pp. 1612-1623, 2008.
[4] H. Zhao, R. Wang, X. Wu et al., "Erythropoietin delivered via intra-arterial infusion reduces endoplasmic reticulum stress in brain microvessels of rats following cerebral ischemia and reperfusion," Journal of Neuroimmune Pharmacology, vol. 10, no. 1, pp. 153-161, 2015.

[5] C. Yao, J. Zhang, G. Liu, F. Chen, and Y. Lin, "Neuroprotection by (-)-epigallocatechin-3-gallate in a rat model of stroke is mediated through inhibition of endoplasmic reticulum stress," Molecular Medicine Reports, vol. 9, no. 1, pp. 69-72, 2014.

[6] Q. Xin, B. Ji, B. Cheng et al., "Endoplasmic reticulum stress in cerebral ischemia," Neurochemistry International, vol. 68, no. 1, pp. 18-27, 2014.

[7] N. Osada, Y. Kosuge, K. Ishige, and Y. Ito, "Characterization of neuronal and astroglial responses to ER stress in the hippocampal CA1 area in mice following transient forebrain ischemia," Neurochemistry International, vol. 57, no. 1, pp. 1-7, 2010.

[8] N. Badiola, C. Penas, A. Minano-Molina et al., "Induction of ER stress in response to oxygen-glucose deprivation of cortical cultures involves the activation of the PERK and IRE-1 pathways and of caspase-12," Cell Death Disease, vol. 2, no. 2, article e149, 2011.

[9] V. P. Nakka, A. Gusain, and R. Raghubir, "Endoplasmic reticulum stress plays critical role in brain damage after cerebral ischemia/reperfusion in rats," Neurotoxicity Research, vol. 17, no. 2, pp. 189-202, 2010.

[10] M. F. Avila, R. Cabezas, D. Torrente et al., "Novel interactions of GRP78: UPR and estrogen responses in the brain," Cell Biology International, vol. 37, no. 6, pp. 521-532, 2013. 
[11] A. Benavides, D. Pastor, P. Santos, P. Tranque, and S. Calvo, "CHOP plays a pivotal role in the astrocyte death induced by oxygen and glucose deprivation," Glia, vol. 52, no. 4, pp. 261$275,2005$.

[12] H. Mamady and K. B. Storey, "Coping with the stress: expression of ATF4, ATF6, and downstream targets in organs of hibernating ground squirrels," Archives of Biochemistry and Biophysics, vol. 477, no. 1, pp. 77-85, 2008.

[13] J. Shen and R. Prywes, "Dependence of site-2 protease cleavage of ATF 6 on prior site-1 protease digestion is determined by the size of the luminal domain of ATF6," The Journal of Biological Chemistry, vol. 279, no. 41, pp. 43046-43051, 2004.

[14] T. H. Sanderson, M. Gallaway, and R. Kumar, "Unfolding the unfolded protein response: unique insights into brain ischemia," International Journal of Molecular Sciences, vol. 16, no. 4, pp. 7133-7142, 2015.

[15] X. Qi, Y. Okuma, T. Hosoi, and Y. Nomura, "Edaravone protects against hypoxia/ischemia-induced endoplasmic reticulum dysfunction," The Journal of Pharmacology and Experimental Therapeutics, vol. 311, no. 1, pp. 388-393, 2004.

[16] H. Hara, "The involvement of endoplasmic reticulum stress in pathogenesis after cerebral ischemia," Clinical Neurology, vol. 50, no. 11, pp. 881-887, 2010.

[17] Q. Yuan, J. Wang, Q.-H. Fang et al., "Attenuating effect of pretreatment with Yiqifumai on lipopolysaccharide-induced intestine injury and survival rate in rat," Journal of Inflammation, vol. 8, no. 1, article 10, 2011.

[18] L. Xing, M. Jiang, L. Dong et al., "Cardioprotective effects of the YiQiFuMai injection and isolated compounds on attenuating chronic heart failure via NF- $\kappa \mathrm{B}$ inactivation and cytokine suppression," Journal of Ethnopharmacology, vol. 148, no. 1, pp. 239-245, 2013.

[19] Y.-Q. Wang, C.-H. Liu, J.-Q. Zhang, D.-N. Zhu, and B.-Y. Yu, "Protective effects and active ingredients of yi-qi-fu-mai sterile powder against myocardial oxidative damage in mice," Journal of Pharmacological Sciences, vol. 122, no. 1, pp. 17-27, 2013.

[20] Y. Tan, F. Li, Y. Lv, K. Zhai, C. Chai, J. Kou et al., "Study on the multi-targets mechanism of YiQiFuMai powder injection on cardio-cerebral ischemic diseases based on network pharmacology," Journal of Proteomics \& Computational Biology, vol. 1, no. 1, pp. 1-9, 2014.

[21] G. S. Cao, X. Y. Ye, Y. Q. Xu et al., "YiQiFuMai powder injection ameliorates blood-brain barrier dysfunction and brain edame after focal cerebral ischemia-reperfusion injury in mice," Drug Design, Development and Therapy, vol. 10, pp. 315-325, 2016.

[22] Y.-Y. Fan, Z. Shen, P. He et al., "A novel neuroprotective strategy for ischemic stroke: transient mild acidosis treatment by $\mathrm{CO}_{2}$ inhalation at reperfusion," Journal of Cerebral Blood Flow \& Metabolism, vol. 34, no. 2, pp. 275-283, 2014.

[23] X. Kong, X. Su, J. Zhu et al., "Neuroprotective effect of buyang huanwu decoction on rat ischemic/reperfusion brain damage by promoting migration of neural precursor cells," Rejuvenation Research, vol. 17, no. 3, pp. 264-275, 2014.

[24] E. Z. Longa, P. R. Weinstein, S. Carlson, and R. Cummins, "Reversible middle cerebral artery occlusion without craniectomy in rats," Stroke, vol. 20, no. 1, pp. 84-91, 1989.

[25] Z. Guo, G. Cao, H. Yang et al., "A combination of four active compounds alleviates cerebral ischemia-reperfusion injury in correlation with inhibition of autophagy and modulation of AMPK/mTOR and JNK pathways," Journal of Neuroscience Research, vol. 92, no. 10, pp. 1295-1306, 2014.
[26] S.-L. Huang, X.-J. He, Z.-F. Li, L. Lin, and B. Cheng, "Neuroprotective effects of ginsenoside Rgl on oxygen-glucose deprivation reperfusion in PC12 cells," Die Pharmazie, vol. 69, no. 3, pp. 208211, 2014.

[27] Y. R. Kim, H. N. Kim, J. Y. Jang et al., "Effects of electroacupuncture on apoptotic pathways in a rat model of focal cerebral ischemia," International Journal of Molecular Medicine, vol. 32, no. 6, pp. 1303-1310, 2013.

[28] K. Shen, Y. Wang, Y. Zhang et al., "Cocktail of four active components derived from Sheng-mai San inhibits hydrogen peroxide induced PC12 cell apoptosis linked with caspase3/ROCK1/MLC pathway," Rejuvenation Research, vol. 18, no. 6, pp. 517-527, 2015.

[29] G. Zeng, S. Zong, Z. Zhang et al., "The role of 6-gingerol on inhibiting Amyloid $\beta$-protein-induced apoptosis in PC12 cells," Rejuvenation Research, vol. 18, no. 5, pp. 413-421, 2015.

[30] F. Li, T. Hayashi, G. Jin et al., "The protective effect of dantrolene on ischemic neuronal cell death is associated with reduced expression of endoplasmic reticulum stress markers," Brain Research, vol. 1048, no. 1-2, pp. 59-68, 2005.

[31] B. Qiu, S. Hu, L. Liu et al., "CART attenuates endoplasmic reticulum stress response induced by cerebral ischemia and reperfusion through upregulating BDNF synthesis and secretion," Biochemical and Biophysical Research Communications, vol. 436, no. 4, pp. 655-659, 2013.

[32] K. Srinivasan and S. S. Sharma, "3-Bromo-7-nitroindazole attenuates brain ischemic injury in diabetic stroke via inhibition of endoplasmic reticulum stress pathway involving CHOP," Life Sciences, vol. 90, no. 3-4, pp. 154-160, 2012.

[33] S.-R. Duan, J.-X. Wang, J. Wang, R. Xu, J.-K. Zhao, and D.-S. Wang, "Ischemia induces endoplasmic reticulum stress and cell apoptosis in human brain," Neuroscience Letters, vol. 475, no. 3, pp. 132-135, 2010.

[34] X. Liu, S. Zhao, F. Liu et al., "Remote ischemic postconditioning alleviates cerebral ischemic injury by attenuating endoplasmic reticulum stress-mediated apoptosis," Translational Stroke Research, vol. 5, no. 6, pp. 692-700, 2014.

[35] S. K. Kwon, M. Ahn, H. J. Song et al., "Nafamostat mesilate attenuates transient focal ischemia/reperfusion-induced brain injury via the inhibition of endoplasmic reticulum stress," Brain Research, vol. 1627, no. 1, pp. 12-20, 2015.

[36] E. Gouix, A. Buisson, A. Nieoullon et al., "Oxygen glucose deprivation-induced astrocyte dysfunction provokes neuronal death through oxidative stress," Pharmacological Research, vol. 87, no. 1, pp. 8-17, 2014.

[37] C. He, A. R. Stroink, and C. X. Wang, "The role of DAPK-BimEL pathway in neuronal death induced by oxygen-glucose deprivation," Neuroscience, vol. 258, no. 1, pp. 254-262, 2014.

[38] T. Urbanek, W. Kuczmik, A. Basta-Kaim, and B. Gabryel, "Rapamycin induces of protective autophagy in vascular endothelial cells exposed to oxygen-glucose deprivation," Brain Research, vol. 1553, no. 1, pp. 1-11, 2014.

[39] A. Kritis, C. Pourzitaki, I. Klagas, M. Chourdakis, and M. Albani, "Proteases inhibition assessment on PC12 and ngf treated cells after oxygen and glucose deprivation reveals a distinct role for aspartyl proteases," PLoS ONE, vol. 6, no. 10, Article ID e25950, 2011.

[40] J. A. Hillion, K. Takahashi, D. Maric, C. Ruetzler, J. L. Barker, and J. M. Hallenbeck, "Development of an ischemic tolerance model in a PC12 cell line," Journal of Cerebral Blood Flow and Metabolism, vol. 25, no. 2, pp. 154-162, 2005. 
[41] B. R. S. Broughton, D. C. Reutens, and C. G. Sobey, "Apoptotic mechanisms after cerebral ischemia," Stroke, vol. 40, no. 5, pp. e331-e339, 2009.

[42] F. Cheng, X. Zhong, Y. Lu et al., "Refined qingkailing protects MCAO mice from endoplasmic reticulum stress-induced apoptosis with a broad time window," Evidence-Based Complementary and Alternative Medicine, vol. 2012, Article ID 567872, 12 pages, 2012.

[43] P. M. Gharibani, J. Modi, C. Pan et al., "The mechanism of taurine protection against endoplasmic reticulum stress in an animal stroke model of cerebral artery occlusion and strokerelated conditions in primary neuronal cell culture," Advances in Experimental Medicine and Biology, vol. 776, no. 1, pp. 241-258, 2013.

[44] X. Y. Zhang, T. T. Zhang, D. D. Song et al., "Endoplasmic reticulum chaperone GRP78 is involved in autophagy activation induced by ischemic preconditioning in neural cells," Molecular Brain, vol. 8, article 20, 2015.

[45] A. V. Kristen, K. Ackermann, S. Buss et al., "Inhibition of apoptosis by the intrinsic but not the extrinsic apoptotic pathway in myocardial ischemia-reperfusion," Cardiovascular Pathology, vol. 22, no. 1, pp. 280-286, 2013.

[46] X. Liu, M. Wang, H. Chen et al., "Hypothermia protects the brain from transient global ischemia/reperfusion by attenuating endoplasmic reticulum response-induced apoptosis through CHOP," PLoS ONE, vol. 8, no. 1, Article ID e53431, 2013.

[47] F. Li, Y. Tan, H. Chen et al., "Identification of schisandrin as a vascular endothelium protective component in YiQiFuMai Powder Injection using HUVECs binding and HPLC-DAD-QTOF-MS/MS analysis," Journal of Pharmacological Sciences, vol. 129, no. 1, pp. 1-8, 2015.

[48] J. Liang, Y. Yu, B. Wang et al., "Ginsenoside Rb1 attenuates oxygen-glucose deprivation-induced apoptosis in SH-SY5Y cells via protection of mitochondria and inhibition of AIF and cytochrome c release," Molecules, vol. 18, no. 10, pp. 12777-12792, 2013.

[49] J. S. Mu, H. Lin, J. X. Ye, M. Lin, and X. P. Cui, "Rg1 exhibits neuroprotective effects by inhibiting the endoplasmic reticulum stressmediated cJun Nterminal protein kinase apoptotic pathway in a rat model of Alzheimer's disease," Molecular Medicine Reports, vol. 12, no. 3, pp. 3862-3868, 2015.

[50] D. Liu, H. Zhang, W. Gu, Y. Liu, and M. Zhang, "Neuroprotective effects of ginsenoside Rbl on high glucose-induced neurotoxicity in primary cultured rat," PLoS ONE, vol. 8, no. 11, Article ID e79399, 2013.

[51] S. S. Cao and R. J. Kaufman, "Endoplasmic reticulum stress and oxidative stress in cell fate decision and human disease," Antioxidants \& Redox Signaling, vol. 21, no. 3, pp. 396-413, 2014.

[52] X. Yang, T. Du, X. Wang et al., "IDH1, a CHOP and C/EBP $\beta$ responsive gene under ER stress, sensitizes human melanoma cells to hypoxia-induced apoptosis," Cancer Letters, vol. 365, no. 2, pp. 201-210, 2015.

[53] E. Dufey, D. Sepúlveda, D. Rojas-Rivera, and C. Hetz, "Cellular mechanisms of endoplasmic reticulum stress signaling in health and disease," American Journal of Physiology-Cell Physiology, vol. 307, no. 7, pp. c582-c594, 2014. 


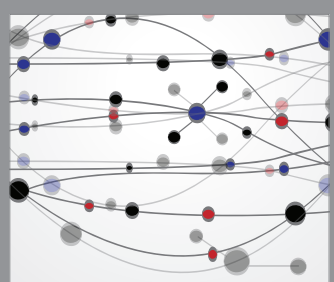

The Scientific World Journal
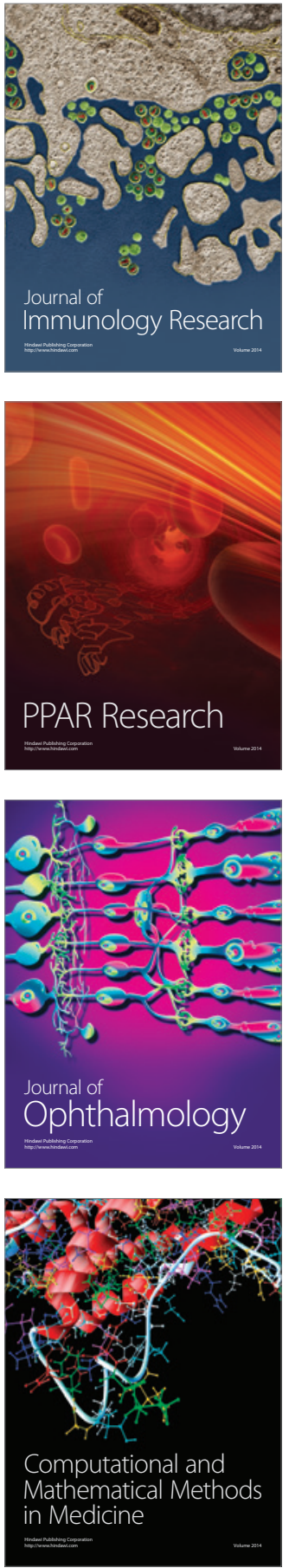

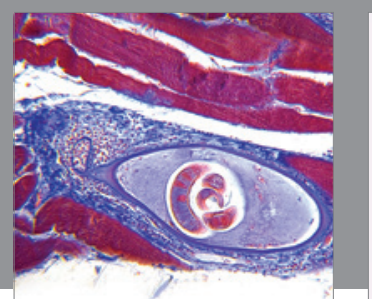

Gastroenterology Research and Practice

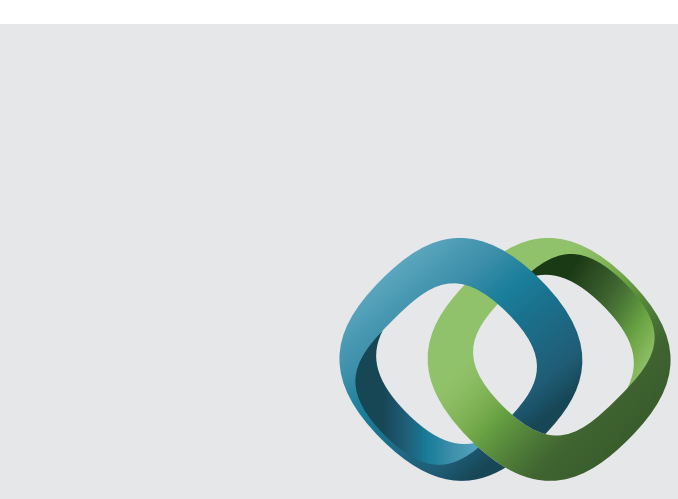

\section{Hindawi}

Submit your manuscripts at

http://www.hindawi.com
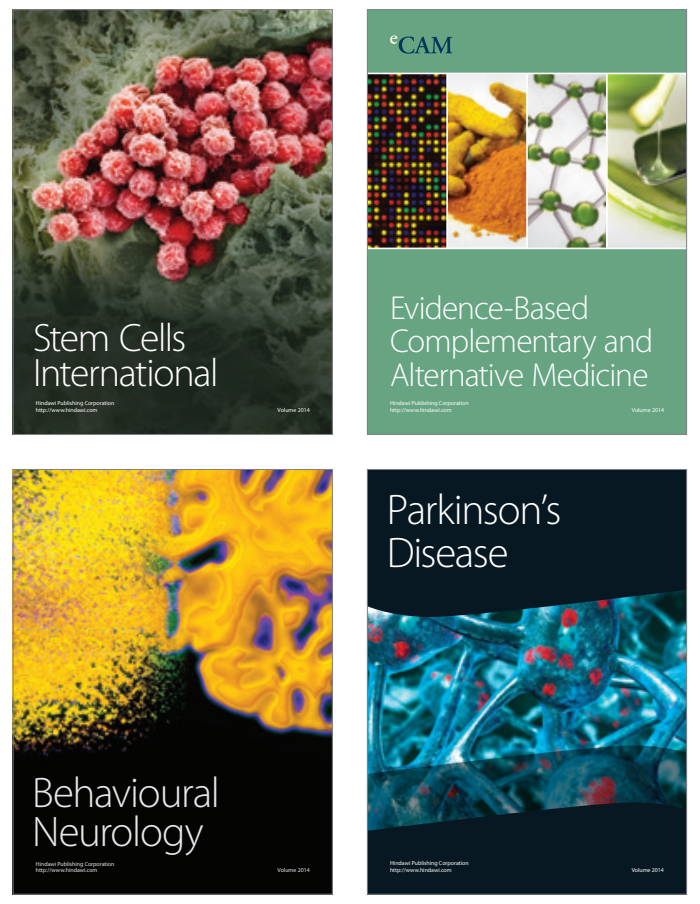
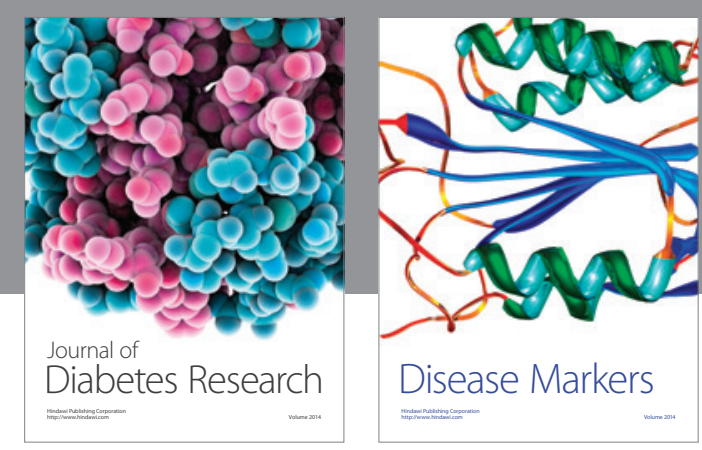

Disease Markers
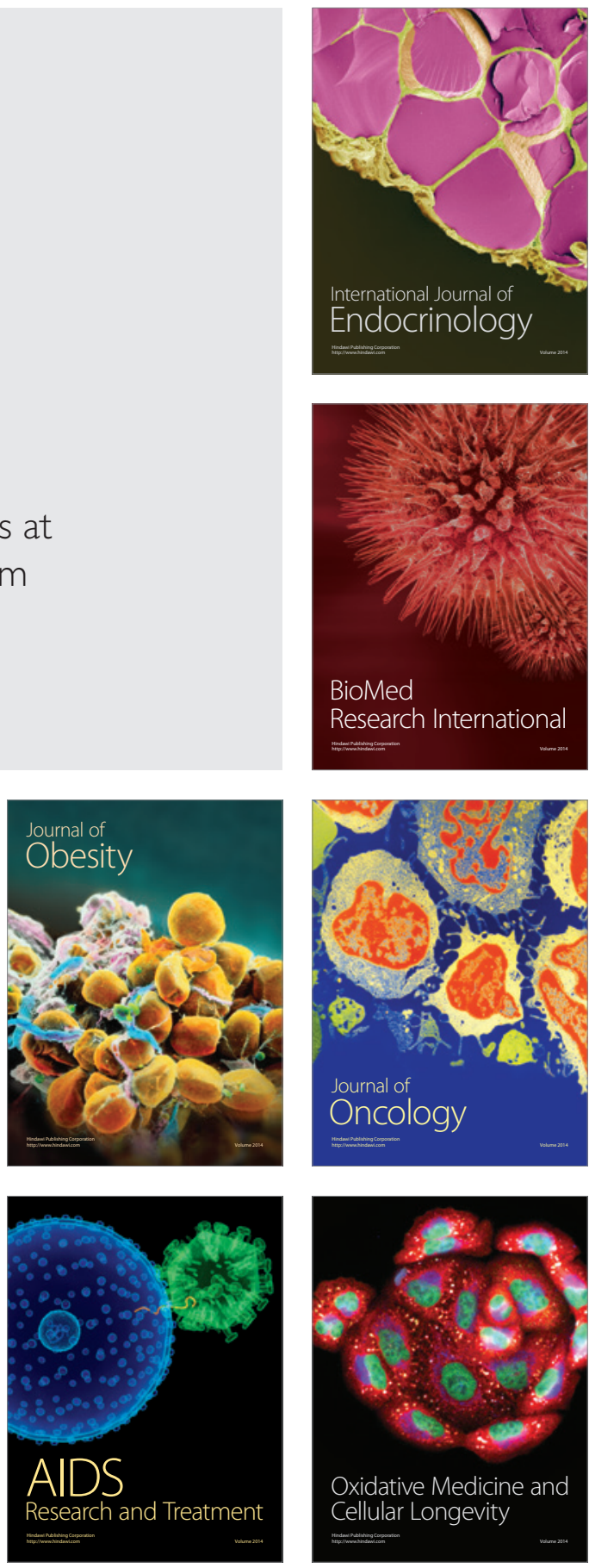